\title{
CROSSED PRODUCTS AND INNER ACTIONS OF HOPF ALGEBRAS
}

\author{
ROBERT J. BLATTNER, MIRIAM COHEN AND SUSAN MONTGOMERY
}

\begin{abstract}
This paper develops a theory of crossed products and inner (weak) actions of arbitrary Hopf algebras on noncommutative algebras. The theory covers the usual examples of inner automorphisms and derivations, and in addition is general enough to include "inner" group gradings of algebras. We prove that if $\pi: H \rightarrow \bar{H}$ is a Hopf algebra epimorphism which is split as a coalgebra map, then $H$ is algebra isomorphic to $A \#_{\sigma} H$, a crossed product of $H$ with the left Hopf kernel $A$ of $\pi$. Given any crossed product $A \#_{\sigma} H$ with $H$ (weakly) inner on $A$, then $A \#_{\sigma} H$ is isomorphic to a twisted product $A_{\tau}[H]$ with trivial action. Finally, if $H$ is a finite dimensional semisimple Hopf algebra, we consider when semisimplicity or semiprimeness of $A$ implies that of $A \#_{\sigma} H$; in particular this is true if the (weak) action of $H$ is inner.
\end{abstract}

Introduction. The purpose of this paper is to begin to lay the foundations of a general theory of actions of Hopf algebras on noncommutative algebras. The importance of such a theory derives from three special cases in which the Hopf algebra is a group algebra, an enveloping algebra of a Lie algebra, or the dual of a group algebra. These cases show that the theory encompasses the study of actions of groups as automorphisms of algebras, the study of actions of Lie algebras as derivations of algebras, and the study of group graded rings, respectively. Each of these areas of study have been quite active lately (see $[\mathbf{1 6}, \mathbf{9}, \mathbf{5}]$ ).

A fundamental concept in the first two cases has been the notion of inner action. One of the major purposes of this paper is to study inner actions of Hopf algebras; as a new example, we will investigate what is meant by an inner grading. Another important concept in the first two cases is that of a semidirect product (smash product): for group actions we have skew group rings and for Lie algebra actions we have differential polynomial rings. This notion is also defined for Hopf algebra actions. More generally one can consider crossed products $A \#_{\sigma} H$ of an algebra $A$ with a Hopf algebra $H$, where the multiplication of the copy of $H$ in $A \#_{\sigma} H$ is twisted by a cocycle $\sigma$, and their study is the other major purpose of this paper. It turns out that these two concepts (inner actions and crossed products) are closely interrelated.

Now inner actions and crossed products of Hopf algebras were both studied by Sweedler [24] in the context of the cohomology theory of Hopf algebras. The present paper owes a great debt to Sweedler's work, especially in $\S \S 1$ and 4 . However, his set-up was restricted at crucial places to the situation where $H$ is a cocommutative

Received by the editors December 12, 1985.

1980 Mathematics Subject Classification (1985 Revision). Primary 16A24; Secondary 16A72, $16 \mathrm{~A} 03,46 \mathrm{~L} 40$.

The third author was partially supported by NSF Grant DMS 8500959 and by a Guggenheim Memorial Foundation Fellowship. 
Hopf algebra and $A$ is an abelian $H$-module algebra. This is inadequate for our purposes, as we now show.

Let $G$ be a group, $N$ a normal subgroup, and $k$ a field. We would like things to be formulated in a general enough way that we can write $k G$ in the form $k N \#_{\sigma} k[G / N]$. Here $k[G / N]$ is our Hopf algebra, $k N$ is the algebra on which it acts, and $\sigma$ is a suitable cocycle. This general crossed product is already an object of study in ring theory $[\mathbf{1 2}, \mathbf{1 8}]$ and has proved useful in studying group rings [14]. It is constructed as follows:

For each $\bar{x} \in \bar{G}=G / N$, choose a coset representative $\gamma(\bar{x}) \in \bar{x}$. For convenience choose $\gamma(\overline{1})=1$. Extending $\gamma$ linearly to $k \bar{G}$ we see that $\gamma$ linearly splits the exact sequence $k G \stackrel{\pi}{\rightarrow} k \bar{G} \rightarrow 0$. Although $\gamma$ is not an algebra homomorphism, it is a coalgebra homomorphism.

Clearly we have $k G=k N \gamma(\bar{G})$. Representing the elements of $k G$ as linear combinations of elements of the form $n \gamma(\bar{x}), n \in N, \bar{x} \in \bar{G}$, we see that they multiply as in a "classical" crossed product:

$$
\begin{aligned}
(n \gamma(\bar{x}))(m \gamma(\bar{y})) & =n\left(\gamma(\bar{x}) m \gamma(\bar{x})^{-1}\right)\left(\gamma(\bar{x}) \gamma(\bar{y}) \gamma(\bar{x} \bar{y})^{-1}\right) \gamma(\bar{x} \bar{y}) \\
& =n(\bar{x} \cdot m) \sigma(\bar{x}, \bar{y}) \gamma(\bar{x} \bar{y})
\end{aligned}
$$

where

$$
\bar{x} \cdot m=(\operatorname{ad} \gamma(\bar{x})) m \text { and } \sigma(\bar{x}, \bar{y})=\gamma(\bar{x}) \gamma(\bar{y}) \gamma(\bar{x} \bar{y})^{-1} .
$$

Since $N$ is normal in $G, k N$ is stable under this action of $\bar{G}$ and $\sigma$ has values in $N$. Extended linearly, this is the multiplication of $k N \#_{\sigma} k \bar{G}$.

Unlike the classical situation, $k N$ is not a $\bar{G}$-module in general, $k N$ is not commutative, and the values of $\sigma$ do not necessarily lie in the center of $N$. Instead, $\bar{G}$ is mapped into Aut $(k N)$ nonhomomorphically (we say $\bar{G}$ acts weakly on $k N$ ) and the cocycle $\sigma$ satisfies a "twisted module" condition:

$$
[\bar{x} \cdot(\bar{y} \cdot n)] \sigma(\bar{x}, \bar{y})=\sigma(\bar{x}, \bar{y})(\bar{x} \bar{y} \cdot n)
$$

for all $\bar{x}, \bar{y} \in \bar{G}$ and $n \in N$. Consequently, $\sigma$ has values central in $N$ if and only if $k N$ is a $\bar{G}$-module.

Thus our general set-up must involve "weak" actions of $H$ on a noncocommutative $A$ (that is, $A$ satisfies all the conditions for being an $H$-module algebra except for being an $H$-module) and cocycles $\sigma$ which take noncommuting values. In addition, in order that the theory be applicable to group gradings, $H$ must be allowed to be noncommutative.

One feature of our approach to inner weak actions already appears in Sweedler: It does not seem to be possible (or desirable) to define what it means for an element of a Hopf algebra to act in an inner fashion, as can be done in the cases of inner automorphisms and inner derivations; instead, it is necessary to speak of inner actions of Hopf subalgebras. We shall see this clearly in our example of inner gradings.

This paper is organized as follows: in $\S 1$, we introduce the basic definitions of weak actions, inner (weak) actions, strongly inner actions, and outer actions, with a number of examples. In $\S 2$, we replace actions by coactions and consider the definitions corresponding to those in $\S 1$. $\S 3$ consists of two examples studied in more depth: inner and outer group gradings, and the semicenter of a Hopf algebra. 
In $\S 4$ we introduce crossed products $A \#_{\sigma} H$. The main result of this section (and perhaps the main result of this paper) is Theorem 4.14 in which it is shown that if $H \stackrel{\pi}{\rightarrow} \bar{H} \rightarrow 0$ is an exact sequence of Hopf algebras with coalgebra splitting, then $H \simeq A \#_{\sigma} \bar{H}$, where $A$ is the left Hopf kernel of $\pi$ and $\sigma$ is a suitable cocycle.

$\S 5$ investigates the interrelationship between inner actions and crossed products: if $H$ is inner on $A$, we see that any crossed product $A \#_{\sigma} H$ is isomorphic to a twisted product $A_{\tau}[H]$ with trivial action; moreover when also $\sigma$ is trivial, $A \# H \cong$ $A \otimes H$ if and only if the action of $H$ is strongly inner. We then consider various natural actions of $H$ or $H^{0}$ on $A \# H$, or $H^{0} \# H$, and determine conditions under which they are inner or outer. In particular it is shown that the RL-condition of Blattner and Montgomery [2] can be reformulated in terms of whether a certain action is inner. Much of the work in this section can be best viewed in terms of nonabelian cohomology of Hopf algebras; however this will be discussed in a subsequent paper.

Finally in $\S 6$ we consider Maschke-type theorems for Hopf algebras. In particular, we apply one of the results of $\S 5$ to prove that if $H$ is a finite-dimensional semisimple Hopf algebra actng on a semiprime ring $A$, such that the action is inner, then $A \# H$ is semiprime. The general question, if $H$ is not inner, remains open.

Throughout we shall follow the notation and conventions of Sweedler's book [25]. In particular, if $H$ is a Hopf algebra, $\Delta$ will denote its comultiplication and $\varepsilon$ its counit. $H^{0}=\left\{f \in H^{*}: f\right.$ vanishes on an ideal of finite codimension $\}$ is also a Hopf algebra [25, Chapter VI]. Consistent use will be made of "sigma notation" (see $[\mathbf{2 5}, \S 1.2]$ ). The letter $k$ will denote a field. If $V$ and $W$ are $k$-vector spaces, $\operatorname{Hom}_{k}(V, W)$ will denote the space of $k$-linear maps from $V$ to $W$ and $\operatorname{End}_{k} V$ will denote $\operatorname{Hom}_{k}(V, V)$. All algebras over $k$ will have a unit 1. If $A$ and $B$ are $k$ algebras, then $\operatorname{Alg}(A, B)$ will denote the space of morphisms of algebras with 1 from $A$ to $B$. The space of isomorphisms in $\operatorname{Alg}(A, A)$ will be denoted by Aut $A$, while the space of $k$-linear derivations of $A$ will be denoted by $\operatorname{Der}_{k} A$. The spaces of inner automorphisms and inner derivations of $A$ will be denoted by $\operatorname{Int} A$ and Int-Der ${ }_{k} A$, respectively.

If $C$ is a coalgebra with counit and $A$ is an algebra, then $\operatorname{Hom}_{k}(C, A)$ becomes an algebra under convolution [25, pp. 69-70]. If $A$ is an algebra and $H$ a Hopf algebra, we can define a map $i: A \otimes H \rightarrow \operatorname{Hom}_{k}\left(H^{0}, A\right)$, by $i(b \otimes h)(g)=\langle g, h\rangle b$ for all $b \in A, h \in H$, and $g \in H^{0}$. We give $\operatorname{Hom}_{k}\left(H^{0}, A\right)$ the finite discrete topology. We leave it to the reader to verify that $\operatorname{Hom}_{k}\left(H^{0}, A\right)$ is a complete topological algebra under convolution, that $i(A \otimes H)$ is dense in $\operatorname{Hom}_{k}\left(H^{0}, A\right)$, and that $i \in \operatorname{Alg}\left(A \otimes H, \operatorname{Hom}_{k}\left(H^{0}, A\right)\right)$.

The first and third authors thank the Mathematical Sciences Research Institute, Berkeley, and the second author thanks the University of Southern California and the University of California, Los Angeles, for their hospitality while this work was done.

1. Inner actions. In this section we discuss some basic notions: weak actions, inner actions, strongly inner actions, and outer actions.

The definition of an inner action of a Hopf algebra $H$ on an algebra $A$ appears in Sweedler's paper [24]. For the applications we treat in $\S 4$, we must generalize his notion somewhat: our weak actions need not turn $A$ into an $H$-module. 
Definition 1.1. Let $H$ be a Hopf algebra and $A$ an algebra. By a weak action of $H$ on $A$ we mean a bilinear map $(h, a) \rightarrow h \cdot a$ of $H \times A \rightarrow A$ such that, for $h \in H, a, b \in A$,

(1) $h \cdot a b=\sum_{(h)}\left(h_{(1)} \cdot a\right)\left(h_{(2)} \cdot b\right)$,

(2) $h \cdot 1=\varepsilon(h) 1$,

(3) $1 \cdot a=a$.

By an action of $H$ on $A$ we mean a weak action such that

(4) $h \cdot(l \cdot a)=h l \cdot a$ for $h, l \in H, a \in A$.

Conditions (1) and (2) say that $H$ measures $A$ [25, p. 139].

DEFinition 1.2. A weak action of $H$ on $A$ is inner if there exists an invertible $u$ in the convolution algebra $\operatorname{Hom}_{k}(H, A)$ such that, for $h \in H$ and $a \in A$,

$$
h \cdot a=\sum_{(h)} u\left(h_{(1)}\right) a u^{-1}\left(h_{(2)}\right),
$$

where $u^{-1}$ is the convolution inverse of $u$. We say that $u$ implements the weak action.

We shall give some standard examples of these notions in a minute. But first we introduce an object that will occur again and again in this paper, and we prove a lemma.

DEFINITION 1.3. Let $u$ be invertible in $\operatorname{Hom}_{k}(H, A)$. Then the bilinear map $t: H \times H \rightarrow A$ is defined by

$$
t(h, l)=\sum_{(h)(l)} u^{-1}\left(l_{(1)}\right) u^{-1}\left(h_{(1)}\right) u\left(h_{(2)} l_{(2)}\right) .
$$

LEMMA 1.4. Let $u$ be invertible in $\operatorname{Hom}_{k}(H, A)$ and set

$$
h \cdot a=\sum_{(h)} u\left(h_{(1)}\right) a u^{-1}\left(h_{(2)}\right)
$$

for $h \in H, a \in A$. Then $u(1)$ is invertible in $A$ and $u(1)^{-1}=u^{-1}(1)$. Moreover, a necessary and sufficient condition that $(h, a) \mapsto h \cdot a$ be a weak action is that $u(1) \in Z(A)$, in which case we have an action if and only if $t(H \times H) \subseteq Z(A)$.

Proof. Conditions (1) and (2) of Definition 1.1 are automatically satisfied for any invertible $u \in \operatorname{Hom}_{k}(H, A)$. Indeed,

$$
\begin{aligned}
h \cdot a b & =\sum_{(h)} u\left(h_{(1)}\right) a b u^{-1}\left(h_{(2)}\right)=\sum_{(h)} u\left(h_{(1)}\right) a u^{-1}\left(h_{(2)}\right) u\left(h_{(3)}\right) b u^{-1}\left(h_{(4)}\right) \\
& =\sum_{(h)}\left(h_{(1)} \cdot a\right)\left(h_{(2)} \cdot b\right),
\end{aligned}
$$

and

$$
h \cdot 1=\sum_{(h)} u\left(h_{(1)}\right) u^{-1}\left(h_{(2)}\right)=\varepsilon(h) 1 .
$$

Clearly, $u(1) u^{-1}(1)=u^{-1}(1) u(1)=\varepsilon(1) 1=1$ so that $u(1)$ is invertible and $u^{-1}(1)=u(1)^{-1}$. Now $1 \cdot a=u(1) a u^{-1}(1)$ so that $(1 \cdot a) u(1)=u(1) a$ for all 
$a \in A$. Thus $u(1) \in Z(A)$ if and only if $1 \cdot a=a$ for all $a \in A$, which is condition (3). Let $h, l \in H, a \in A$. Then

$$
h l \cdot a=\sum_{(h)(l)} u\left(h_{(1)} l_{(1)}\right) a u^{-1}\left(h_{(2)} l_{(2)}\right)
$$

while

$$
h \cdot(l \cdot a)=\sum_{(h)(l)} u\left(h_{(1)}\right) u\left(l_{(1)}\right) a u^{-1}\left(l_{(2)}\right) u^{-1}\left(h_{(2)}\right) .
$$

Thus condition (4) holds if and only if

$$
t(h, l)=\sum_{(h)(l)} u^{-1}\left(l_{(1)}\right) u^{-1}\left(h_{(1)}\right) u\left(h_{(2)} l_{(2)}\right)
$$

commutes with all $a \in A$ for all $h, l \in H$.

We now turn to our examples.

EXAMPLE 1.5. The trivial action of $H$ on $A$ is given by $h \cdot a=\varepsilon(h) a$ for $h \in H, a \in A$. The trivial action is always inner: set $u(h)=\varepsilon(h) 1 \in A$ for $h \in H$. Conversely, if $A$ is any commutative algebra, then any inner weak action of $H$ on $A$ is trivial.

EXAMPLE 1.6. Let $G$ be a group and $A$ an algebra, and set $H=k G$. Since $G$ is a basis for $H$, a bilinear map $(h, a) \mapsto h \cdot a$ of $H \times A \rightarrow A$ determines and is determined by a map $\alpha: G \rightarrow \operatorname{End}_{k} A$ by $\alpha(x) a=x \cdot a$ for $x \in G, a \in A$. It is easy to see that the bilinear map is a weak action if and only if $\alpha(G) \subseteq \operatorname{Alg}(A, A)$ and $\alpha(1)=$ id. We claim that a weak action of $H$ is inner if and only if $\alpha(G) \subseteq \operatorname{Int} A$. In fact, a map $u \in \operatorname{Hom}_{k}(H, A)$ is determined by its values on $G$ and any map from $G$ to $A$ extends uniquely to such a $u$. If $u, v \in \operatorname{Hom}_{k}(H, A)$, then $(u * v)(x)=u(x) v(x)$ for $x \in G$, since $\Delta x=x \otimes x$. Thus $u$ is invertible in $\operatorname{Hom}_{k}(H, A)$ if and only if $u(x)$ is invertible in $A$ for all $x \in G$, and then $u^{-1}(x)=u(x)^{-1}$. Finally,

$$
\sum_{(x)} u\left(x_{(1)}\right) a u^{-1}\left(x_{(2)}\right)=u(x) a u(x)^{-1} \quad \text { for } x \in G, a \in A .
$$

From this the claim follows easily.

EXAMPLE 1.7. Let $L$ be a Lie algebra and $H=U(L)$ its enveloping algebra, and let $A$ be an algebra. A bilinear map $(h, a) \mapsto h \cdot a$ of $H \times A \rightarrow A$ determines a linear $\operatorname{map} \delta: L \rightarrow \operatorname{Hom}_{k}(A, A)$ by $\delta(x) a=x \cdot a$ for $x \in L, x \in A$. Since $\Delta x=x \otimes 1+1 \otimes x$ for $x \in L$, we see that, if the bilinear map is a weak action, then $\delta(L) \subseteq \operatorname{Der}_{k} A$. Conversely, suppose that we are given a linear map $\delta: L \rightarrow \operatorname{Der}_{k} A$. We claim that there exists a (generally nonunique) weak action $(h, \alpha) \mapsto h \cdot a$ of $H$ on $A$ such that $\delta$ arises from it as above. In fact, let $\left\{x_{\alpha}\right\}$ be an ordered basis of $L$. Then the ordered monomials $\left\{x_{\alpha_{1}} \cdots x_{\alpha_{n}}: \alpha_{1} \leq \alpha_{2} \leq \cdots \leq \alpha_{n}, 0 \leq n \in \mathbf{Z}\right\}$ form a linear basis of $H$. Set $\delta\left(x_{\alpha_{1}} \cdots x_{\alpha_{n}}\right)=\delta\left(x_{\alpha_{1}}\right) \cdots \delta\left(x_{\alpha_{n}}\right) \in \operatorname{End}_{k} A$ for $\alpha_{1} \leq \alpha_{2} \leq \cdots \leq \alpha_{n}$, extend $\delta$ to a linear map of $H$ to $\operatorname{End}_{k} A$, and set $h \cdot a=\delta(h) a$ for $h \in H, a \in A$. Since $\Delta$ is an algebra homomorphism and since $\varepsilon\left(x_{\alpha_{1}} \cdots x_{\alpha_{n}}\right)=0$ if $n \geq 1$, it is straightforward to check that $(h, a) \mapsto h \cdot a$ is a weak action.

Suppose that $\delta(L) \subseteq \operatorname{Int}^{-D_{e r}}(A)$. Then there exists a (nonunique!) linear $u: L \rightarrow A$ such that $\delta(x) a=[u(x), a]$ for $x \in L, a \in A$. Extend $u$ to $H$ by setting $u\left(x_{\alpha_{1}} \cdots x_{\alpha_{n}}\right)=u\left(x_{\alpha_{1}}\right) \cdots u\left(x_{\alpha_{n}}\right)$ for $\alpha_{1} \leq \alpha_{2} \leq \cdots \leq \alpha_{n}$. Then it is 
straightforward to check that $u$ is invertible in $\operatorname{Hom}_{k}(H, A)$, that $u^{-1}\left(x_{\alpha_{1}} \cdots x_{\alpha_{n}}\right)=$ $(-1)^{n} u\left(x_{\alpha_{n}}\right) \cdots u\left(x_{\alpha_{1}}\right)$, and that $h \cdot a=\sum_{(h)} u\left(h_{(1)}\right) a u^{-1}\left(h_{(2)}\right)$ for $a \in A$ and $h=x_{\alpha_{1}} \cdots x_{\alpha_{n}}$ with $\alpha_{1} \leq \cdots \leq \alpha_{n}$, where $h \cdot a$ is defined above. The same then holds for all $h \in H$ by linearity. Conversely, suppose the weak action of $H$ on $A$ is inner and is implemented by $u \in \operatorname{Hom}_{k}(H, A)$. Then

$$
x \cdot a=u(x) a u^{-1}(1)+u(1) a u^{-1}(x)=u(x) u(1)^{-1} a+a u(1) u^{-1}(x)
$$

for $x \in L, a \in A$, because $u^{-1}(1)=u(1)^{-1}$ and $u(1) \in Z(A)$ by (1.4). Setting $a=1$, we get $u(x) u(1)^{-1}+u(1) u^{-1}(x)=x \cdot 1=\varepsilon(x) 1=0$. Therefore $x \cdot a=\left[u(x) u(1)^{-1}, a\right]$ so that $\delta(L) \subseteq \operatorname{Int}_{-D^{-}}(A)$.

EXAMPLE 1.8. Any Hopf algebra $H$ acts on itself via the adjoint action [11], defined by $(\operatorname{ad} h) k=\sum_{(h)} h_{(1)} k\left(S h_{(2)}\right)$. This extends the usual notions for $H=$ $k G$, where $(\operatorname{ad} x) y=x y x^{-1}$ for $x, y \in G$; and for $H=U(L)$, where $(\operatorname{ad} x) h=$ $x h-h x$ for $x \in L, h \in H$. Clearly this is an inner action: set $u=\mathrm{id}_{H}$.

EXAMPLE 1.9. Any action of $H$ on $A$ becomes inner on $A \# H$ : set $u(h)=$ $1 \# h$ for $h \in H$. Then $u^{-1}(h)=1 \# S h$ and the weak action of $H$ on $A \# H$ defined by $u$ as in Lemma 1.4 takes the form

$$
\begin{aligned}
h \cdot(a \# l) & =\sum_{(h)}\left(1 \# h_{(1)}\right)(a \# l)\left(1 \# S h_{(2)}\right) \\
& =\sum_{(h)}\left(h_{(1)} \cdot a\right) \#\left(h_{(2)} l S h_{(3)}\right) \\
& =\sum_{(h)}\left(h_{(1)} \cdot a\right) \#\left(\operatorname{ad} h_{(2)}\right) l .
\end{aligned}
$$

In particular $h \cdot(a \# 1)=(h \cdot a) \# 1$. It follows from Lemma 1.16 below that this weak action of $H$ on $A \# H$ is actually an action.

For some purposes it is convenient to reformulate Definition 1.2 as follows: For each $a \in A$, define $\hat{a} \in \operatorname{Hom}_{k}(H, A)$ by setting

$$
\hat{a}(h)=\varepsilon(h) a .
$$

The map $a \mapsto \hat{a}$ is an injective homomorphism of algebras with 1 . Then the formula in (1.2) is just

$$
h \cdot a=\left(u * \hat{a} * u^{-1}\right)(h) .
$$

DEFINITION 1.12. Let $u, v \in \operatorname{Hom}_{k}(H, A)$ be invertible and satisfy $u(1), v(1) \in$ $Z(A)$. We say $u$ is equivalent to $v$, written $u \sim v$, if $u$ and $v$ induce the same weak action of $H$ on $A$ as in Lemma 1.4 .

The next lemma describes the notion of equivalence precisely.

LEMMA 1.13. (1) Let $u, v$ be as in (1.12). Then $u \sim v$ if and only if $v^{-1} * u \in$ $\operatorname{Hom}_{k}(H, Z(A))$.

(2) Let $u$ be as in (1.12). Then there exists $v$ as in (1.12) such that $u \sim v$ and $v(1)=1$.

PROOF. (1) Using (1.11), we see that $u \sim v$ if and only if $u * \hat{a} * u^{-1}=v * \hat{a} * v^{-1}$ for all $a \in A$ if and only if $\left(v^{-1} * u\right) * \hat{a}=\hat{a} *\left(v^{-1} * u\right)$ for all $a \in A$. But this is the same as $\left(v^{-1} * u\right)(h) a=a\left(v^{-1} * u\right)(h)$ for all $a \in A, h \in H$.

(2) Let $v=u * \widehat{u^{-1}(1)}$ and apply Lemma 1.4, part (1) above. 
Lemmas 1.4 and 1.13 say that the inner weak actions of $H$ on $A$ are in natural bijective correspondence with the right cosets $\mathcal{G} / \mathcal{G}_{Z}$, where $\mathcal{G}$ consists of the invertible $u \in \operatorname{Hom}_{k}(H, A)$ such that $u(1)=1$ and $\mathscr{G}_{Z}=\{u \in \mathcal{G}: u(H) \subseteq Z(A)\}$.

We require one more technical lemma. The proof is easy and is the same as in Sweedler [24]; hence we omit it.

LEMMA 1.14. Let $(h, a) \mapsto h \cdot a$ be a weak action of $H$ on $A$. Then the following are equivalent:

(1) the weak action is inner,

(2) there exists an invertible $u \in \operatorname{Hom}_{k}(H, A)$ such that

$$
u(h) a=\sum_{(h)}\left(h_{(1)} \cdot a\right) u\left(h_{(2)}\right), \quad \text { all } h \in H, a \in A ;
$$

(3) there exists an invertible $v \in \operatorname{Hom}_{k}(H, A)$ such that

$$
a v(h)=\sum_{(h)} v\left(h_{(1)}\right)\left(h_{(2)} \cdot a\right), \quad \text { all } h \in H, a \in A .
$$

Moreover, $u$ or $v^{-1}$ implements the inner weak action.

Definition 1.15. A weak action of $H$ on $A$ is strongly inner if it can be implemented by some $u \in \operatorname{Alg}(H, A)$.

LemMa 1.16. Let $u \in \operatorname{Alg}(H, A)$. Then $u$ is convolution invertible in $\operatorname{Hom}_{k}(H, A), u^{-1}=u \circ S$ (so in particular $u^{-1}$ is an algebra antihomomorphism), and the weak action implemented by $u$ is in fact an action.

PROOF. Since $u$ is an algebra homomorphism and since $S$ is defined as the convolution inverse of $\operatorname{id}_{H}$ in $\operatorname{End}_{k} H$, it follows that $u \circ S$ is the convolution inverse of $u$ in $\operatorname{Hom}_{k}(H, A)$. We compute, as in (1.4),

$$
\begin{aligned}
t(h, l) & =\sum_{(h)(l)} u\left(S\left(l_{(1)}\right)\right) u\left(S\left(h_{(1)}\right)\right) u\left(h_{(2)} l_{(2)}\right) \\
& =u\left(\sum_{(h)(l)} S\left(l_{(1)}\right) S\left(h_{(1)}\right) h_{(2)} l_{(2)}\right) \\
& =\varepsilon(h) \varepsilon(l) u(1) \in Z(A) .
\end{aligned}
$$

Thus our weak action is an action.

We return to Examples (1.5)-(1.9). The trivial action, the adjoint action, and the action of (1.9) are all strongly inner. In (1.6), if $\alpha(G) \subseteq \operatorname{Int} A$, the corresponding weak action of $k G$ is strongly inner precisely if it can be implemented by a $u$ which is (the linear extension of) a homomorphism of $G$ into the group of invertible elements of $A$.

Suppose the weak action in (1.7) of $U(L)$ on $A$ is strongly inner. It is then an action, whence $\delta$ is a Lie algebra homomorphism of $L$ into $\operatorname{Der}_{k} A$. The action may be implemented by some $u \in \operatorname{Alg}(U(L), A)$. Then $\left.u\right|_{L}$ is a Lie algebra homomorphism of $L$ into $A$ (under commutation). Conversely, suppose $u: L \rightarrow A$ is a Lie algebra homomorphism. Then the extension of $u$ to $U(L)$ given in (1.7) belongs to $\operatorname{Alg}(U(L), A)$. Defining $\delta(x) a=[u(x), a], x \in L, a \in A$, we see that $\delta$ is a Lie homomorphism of $L$ into $\operatorname{Der}_{k} A$. So extending $\delta$ to $U(L)$ as in the first paragraph 
of (1.7), we see that the action of $U(L)$ on $A$ corresponding to $\delta$ is strongly inner. Note that the extensions of $u$ and $\delta$ to $U(L)$ are basis independent in this case, unlike in the more general situation of (1.7).

It is easy to give examples of inner actions which are not strongly inner.

EXAMPLE 1.17. Let $A=C_{2}(k)$, the Clifford algebra over a field $k$ of characteristic not 2 generated by 1 and by $e_{1}, e_{2}$ such that $e_{1}^{2}=e_{2}^{2}=-1$ and $e_{1} e_{2}+e_{2} e_{1}=0$. Let $\mathbf{Z}_{2}=\{1, x\}$ act as automorphisms of $A$ via $x \cdot a=-e_{2} a e_{2}, a \in A$. The action of $k \mathbf{Z}_{2}$ so determined is inner: set $u(1)=1, u(x)=e_{2}$. If the action were strongly inner, then $u$ would be equivalent to an algebra homomorphism $v$, so $v(x)=\lambda e_{2}$ for some $\lambda \in Z(A)$, and $1=v(1)=v\left(x^{2}\right)=\left(\lambda e_{2}\right)^{2}=-\lambda^{2}$. But $Z(A)=k 1$. Thus $k$ would have to contain a square root of -1 . And this is sufficient.

More generally, any inner $\mathbf{Z}_{2}$-action on an algebra $A$ is strongly inner if $Z(A)$ is closed under square roots. This is false for groups other than $\mathbf{Z}_{2}$, as the next example shows.

EXAMPLE 1.18. Let $A=C_{2}(k)$, as in (1.17). Let $G=\mathbf{Z}_{2} \times \mathbf{Z}_{2}=\{1, x, y, x y\}$ with $x^{2}=y^{2}=1, x y=y x$, act as inner automorphisms of $A$ via $x \cdot a=-e_{2} a e_{2}$, $y \cdot a=-e_{1} a e_{1}, a \in A$; that is $u(1)=1, u(x)=e_{2}, u(y)=e_{1}, u(x y)=e_{1} e_{2}$. If this action were strongly inner, then $u \sim v$, an algebra homomorphism. Then $v(x)=\lambda e_{2}, v(y)=\mu e_{1}$ with $\lambda, \mu \in k$, and $v(x) v(y)=v(x y)=v(y x)=v(y) v(x)$. But this implies $\lambda \mu e_{2} e_{1}=\lambda \mu e_{1} e_{2}$, which is false since char $k \neq 2$.

Since a strongly inner weak action of $H$ on $A$ is actually an action (1.16), $A$ is an $H$-module algebra, and we can form $A \# H$. The next result shows that $A \# H$ can be trivialized. It is actually a special case of Theorem 5.3; however the present argument is so much simpler that it is worth doing now (cf. Corollary 5.5).

Proposition 1.19. Suppose $H$ acts on $A$ in a strongly inner way. Then $A \# H \simeq A \otimes_{k} H$, as algebras with 1 .

Proof. Let $u \in \operatorname{Alg}(H, A)$ implement the given action. Define

$$
\phi: A \# H \rightarrow A \otimes_{k} H \quad \text { by } \phi(a \# h)=\sum_{(h)} a u\left(h_{(1)}\right) \otimes h_{(2)} .
$$

Then $\phi$ has an inverse

$$
\psi: A \otimes_{k} H \rightarrow A \# H \quad \text { given by } \quad \psi(a \otimes h)=\sum_{(h)} a u^{-1}\left(h_{(1)}\right) \# h_{(2)},
$$

so it suffices to check that $\phi$ is a homomorphism:

$$
\begin{aligned}
\phi((a \# h)(b \# l)) & =\phi\left(\sum_{(h)} a\left(h_{(1)} \cdot b\right) \# h_{(2)} l\right) \\
& =\sum_{(h)(l)} a\left(h_{(1)} \cdot b\right) u\left(h_{(2)} l_{(1)}\right) \otimes h_{(3)} l_{(2)} \\
& =\sum_{(h)(l)} a u\left(h_{(1)}\right) b u^{-1}\left(h_{(2)}\right) u\left(h_{(3)}\right) u\left(l_{(1)}\right) \otimes h_{(4)} l_{(2)} \\
& =\sum_{(h)(l)} a u\left(h_{(1)}\right) b u\left(l_{(1)}\right) \otimes h_{(2)} l_{(2)} \\
& =\phi(a \# h) \phi(b \# l) .
\end{aligned}
$$


We will prove a converse to this result in Corollary 5.5.

The last important concept in this section is that of an outer weak action.

Definition 1.20. A weak action of $H$ on $A$ is outer if $H$ is not trivial and if, for every Hopf subalgebra $L$ of $H$, the induced weak action of $L$ on $A$ is inner only if $L$ is trivial.

In Example 1.6, a weak action of $H=k G$ on $A$ is outer if and only if $G \neq\{1\}$ and the only subgroup of $G$ contained in $\alpha^{-1}(\operatorname{Int} A)$ is $\{1\}$. This is simply because the Hopf subalgebras of $k G$ are just the $k G_{1}$ for $G_{1}$ a subgroup of $G$. If the weak action of $H$ on $A$ is an action, then $\alpha$ is a homomorphism of $G$ into Aut $A$ and so the action is outer if and only if $\alpha^{-1}(\operatorname{Int} A)=\{1\}$, and we recover the normal notion of outer group action.

In Example 1.7, the situation is trickier in general. So we restrict attention to an action of $H=U(L)$ on $A$ with $L \neq\{0\}$, and suppose that char $k=0$. Then the action is outer if and only if no nonzero Lie subalgebra $L_{1}$ of $L$ acts as inner derivations of $A$. In fact, in characteristic zero, the Hopf subalgebras of $U(L)$ are just the $U\left(L_{1}\right)$ for $L_{1}$ a Lie subalgebra of $L$. Suppose that the action of $H$ restricted to $U\left(L_{1}\right)$ is inner. Then we already know that $L_{1}$ acts by inner derivations. Conversely, if the action of $H$ restricted to $L_{1}$ is by inner derivations, then the construction of $u \in \operatorname{Hom}_{k}\left(U\left(L_{1}\right), A\right)$ of paragraph two of Example 1.7 shows that the action of $H$ restricted to $U\left(L_{1}\right)$ is inner. The situation for a weak action of $H$ on $A$ is unclear, even in characteristic zero.

Let $A$ be an $H$-module algebra. Recall [25] that $A \# H$ becomes an $H^{0}$-module algebra by setting

$$
f \cdot(a \# h)=a \#(f \rightarrow h)=\sum_{(h)} a \#\left\langle f, h_{(2)}\right\rangle h_{(1)}
$$

for $f \in H^{0}, a \in A, h \in H$. Our last example in this section gives conditions under which this action is outer.

THEOREM 1.22. If the action of $H$ on $A$ is strongly inner, then the action of $H^{0}$ on $A \# H$ is outer if $H^{0}$ is not trivial.

PROOF. We first prove the theorem for the special case when the action of $H$ on $A$ is trivial (Example 1.5). In this case $A \# H=A \otimes_{k} H$.

Let $L$ be a Hopf subalgebra of $H^{0}$ and suppose the action of $L$ on $A \otimes H$ is inner. Then there exists an invertible $\bar{u} \in \operatorname{Hom}_{k}\left(L, A \otimes_{k} H\right)$ so that

$$
a \otimes \sum_{(h)}\left\langle f, h_{(2)}\right\rangle h_{(1)}=f \cdot(a \otimes h)=\sum_{(f)} \bar{u}\left(f_{(1)}\right)(a \otimes h) \bar{u}^{-1}\left(f_{(2)}\right)
$$

for all $f \in L^{0}, a \in A, h \in H$. Apply the homomorphism $\bar{\varepsilon}=\mathrm{id} \otimes \varepsilon$ to this equation and set $a=1$. Then

$$
\bar{\varepsilon}\left(1 \otimes \sum_{(h)}\left\langle f, h_{(2)}\right\rangle h_{(1)}\right)=\sum_{(h)}\left\langle f, h_{(2)}\right\rangle \varepsilon\left(h_{(1)}\right) 1=\langle f, h\rangle 1
$$


whereas

$$
\begin{aligned}
\left.\bar{\varepsilon}\left(\sum_{(f)} \bar{u}\left(f_{(1)}\right)(1 \otimes h) \bar{u}^{-1}\left(f_{(2)}\right)\right)=\varepsilon(h) \sum_{(f)} \bar{\varepsilon}\left(\bar{u}\left(f_{(1)}\right)\right) 1 \bar{\varepsilon}^{-1}\left(\bar{u}_{(2)}\right)\right) \\
=\varepsilon(h) \bar{\varepsilon}\left(\sum_{(f)} \bar{u}\left(f_{(1)}\right) \bar{u}^{-1}\left(f_{(2)}\right)\right)=\varepsilon(h) \bar{\varepsilon}(\langle f, 1\rangle 1 \otimes 1) \\
=\varepsilon(h)\langle f, 1\rangle 1 .
\end{aligned}
$$

Thus $f=\langle f, 1\rangle \varepsilon$ so that $L$ is trivial. This shows that the action of $H^{0}$ on $A \otimes H$ is outer.

For the general case, let the strongly inner action of $H$ on $A$ be implemented by $u \in \operatorname{Alg}(H, A)$. By Proposition 1.19, $\phi: A \# H \rightarrow A \otimes_{k} H$ is an isomorphism of algebras, where $\phi(a \# h)=\sum_{(h)} a u\left(h_{(1)}\right) \otimes h_{(2)}$. The theorem will follow if we show that $\phi$ is an $H^{0}$-module morphism. In fact,

$$
\begin{aligned}
\phi(f \cdot(a \# h)) & =\phi\left(\sum_{(h)} a \#\left\langle f, h_{(2)}\right\rangle h_{(1)}\right)=\sum_{(h)}\left\langle f, h_{(3)}\right\rangle a u\left(h_{(1)}\right) \otimes h_{(2)} \\
& =f \cdot\left(\sum_{(h)} a u\left(h_{(1)}\right) \otimes h_{(2)}\right)=f \cdot \phi(a \# h)
\end{aligned}
$$

for $f \in H^{0}, a \in A, h \in H$, as desired.

2. Coactions. In this section we consider weak, inner, strongly inner, and outer coactions of a Hopf algebra $H$ on $A$, and relate these notions to the corresponding notions of $\S 1$.

Just as a weak action of $H$ on $A$ turns $A$ into an $H$-module algebra, except for the condition that $A$ be an $H$-module, so a weak coaction of $H$ on $A$ turns $A$ into an $H$-comodule algebra, except for the condition that $A$ be an $H$-comodule. More precisely, we have

DEFINITION 2.1. Let $H$ be a Hopf algebra and $A$ an algebra. By a weak coaction of $H$ on $A$ we mean a linear map $\rho: A \rightarrow A \otimes_{k} H$ such that, for $a, b \in A$,

(1) $\rho(a b)=\rho(a) \rho(b)$,

(2) $\rho(1)=1 \otimes 1$,

(3) $(\mathrm{id} \otimes \varepsilon) \rho(a)=a$.

(Conditions (1) and (2) merely say $\rho \in \operatorname{Alg}(A, A \otimes H)$.) By a coaction of $H$ on $A$ we mean a weak coaction such that

(4) $(\rho \otimes \mathrm{id}) \circ \rho=(\mathrm{id} \otimes \Delta) \circ \rho$.

Each of the four conditions of Definition 2.1 is the formal dual of the correspondingly numbered condition of Definition 1.1. The following notions are each formal duals of the corresponding notions for weak actions.

DEFinition 2.2. Let $\rho$ be a weak coaction of $H$ on $A$.

(1) $\rho$ is inner if there exists an invertible $s \in A \otimes H$ such that $\rho(a)=s(a \otimes 1) s^{-1}$ for all $a \in A$. We say that $s$ implements $\rho$.

(2) $\rho$ is strongly inner if there exists $s$ as in (1) so that

$$
\mu(s \otimes s)=(\mathrm{id} \otimes \Delta) s,
$$


where $\mu: A \otimes H \otimes A \otimes H \rightarrow A \otimes H \otimes H$ is defined by

$$
\mu(a \otimes h \otimes b \otimes l)=a b \otimes h \otimes l .
$$

(3) $\rho$ is outer if $H$ is not trivial and if, whenever $\pi: H \rightarrow \bar{H}$ is a surjective Hopf algebra morphism such that the induced weak $\bar{H}$-coaction $\bar{\rho}=(\mathrm{id} \otimes \pi) \circ \rho$ is inner, then $\bar{H}$ is trivial.

EXAMPLE 2.3. The trivial coaction of $H$ on $A$ is defined by $\rho(a)=a \otimes 1$. It is strongly inner. For we may let $s=1_{A} \otimes 1_{H}$ and then $\mu(s \otimes s)=1_{A} \otimes 1_{H} \otimes 1_{H}=$ $(\mathrm{id} \otimes \Delta) s$.

EXAMPLE 2.4. The natural coaction $\rho=\Delta$ of $H$ on itself is outer if $H$ is not trivial. In fact, let $\pi: H \rightarrow \bar{H}$ be a surjective Hopf algebra map, and suppose that $\bar{\rho}$ is inner. Then there exists $s \in H \otimes \bar{H}$ such that

$$
\sum_{(h)} h_{(1)} \otimes \pi h_{(2)}=\bar{\rho} h=s(h \otimes \overline{1}) s^{-1} \quad \text { for all } h \in H .
$$

Apply the homomorphism $\theta=\varepsilon \otimes$ id to $H \otimes \bar{H}$. We get

$$
\sum_{(h)} \varepsilon\left(h_{(1)}\right) \pi h_{(2)}=(\theta s)[\varepsilon(h) \overline{1}]\left(\theta s^{-1}\right)=\varepsilon(h) \overline{1},
$$

so that $\pi h=\varepsilon(h) \overline{1}$ for all $h \in H$. Since $\pi$ is surjective, this says that $\bar{H}$ is trivial, as desired. Note that this proof is simply a transcription of the first part of the proof of Theorem 1.21.

Now any weak coaction of $H$ on $A$ determines a weak action of $H^{0}$ on $A$ by setting, for $f \in H^{0}, a \in A$,

$$
f \cdot a=i(\rho(a))(f),
$$

where $i$ is the map from $A \otimes H$ to $\operatorname{Hom}_{k}\left(H^{0}, A\right)$ mentioned in the Introduction. That conditions (1) $-(3)$ of Definition 2.1 for $\rho$ imply conditions (1)-(3) of Definition 1.1 for $(f, a) \rightarrow f \cdot a$ is left to the reader. For ease of notation, we will write $\bar{s}$ for $i(s), s \in A \otimes H$, in what follows.

Of course, $H^{0}$ can be very small (see Lemma 2.7 below). The map $i$ is injective precisely when $H^{0}$ is dense in $H^{*}$ or, equivalently, whenever $H$ is residually finite dimensional (see $[\mathbf{2}, \S 1]$ ). If $H$ is finite dimensional, then $i$ is a bijection.

PROPOSITION 2.6. Let $\rho$ be a weak coaction of $H$ on $A$, and consider the corresponding weak action of $H^{0}$ on $A$, as above.

(1) If $\rho$ is inner, implemented by $s \in A \otimes H$, then the weak action of $H^{0}$ is inner, implemented by $\bar{s} \in \operatorname{Hom}_{k}\left(H^{0}, A\right)$.

(2) If $\rho$ is strongly inner, implemented by $s$ as in Definition 2.2(2), then $\bar{s} \in$ $\operatorname{Alg}\left(H^{0}, A\right)$ and so the $H^{0}$ action is strongly inner.

(3) Suppose $H$ is finite dimensional. Then the converse of (1) and (2) hold. Moreover, $\rho$ is outer if and only if the weak action of $H^{*}$ is outer.

PROOF. (1) By hypothesis, $\rho(a)=s(a \otimes 1) s^{-1}$ and so $\overline{\rho(a)}=\bar{s} * \overline{(a \otimes 1)} * \bar{s}^{-1}$, since $i$ is an algebra homomorphism. In the notation of $(1.10), \overline{a \otimes 1}=\hat{a}$, with $H^{0}$ replacing $H$. Thus $f \cdot a=\overline{\rho(a)}(f)=\left(\bar{s} * \hat{a} * \bar{s}^{-1}\right)(f)$ and so the weak $H^{0}$-action is inner by (1.11). 
(2) We must check that $\bar{s}(f g)=\bar{s}(f) \bar{s}(g)$ for all $f, g \in H^{0}$ and that $\bar{s}(1)=1$. Let $\Phi: A \otimes H \otimes H \rightarrow \operatorname{Hom}_{k}\left(H^{0} \otimes H^{0}, A\right)$ be defined by

$$
\Phi(a \otimes h \otimes l)(f \otimes g)=\langle f, h\rangle\langle g, l\rangle a .
$$

Now

$$
\overline{(a \otimes h)}(f g)=\langle h, f g\rangle a=\langle\Delta h, f \otimes g\rangle a
$$

so that $\Phi((\mathrm{id} \otimes \Delta) s)(f \otimes g)=\bar{s}(f g)$, while $\overline{(a \otimes h)}(f) \overline{(b \otimes l)}(g)=\langle f, h\rangle\langle g, h\rangle a b$ so that

$$
\Phi(\mu(s \otimes s))(f \otimes g)=\bar{s}(f) \bar{s}(g), \quad \text { for all } s \in A \otimes H .
$$

Thus $\mu(s \otimes s)=($ id $\otimes \Delta) s$ implies that $\bar{s}(f) \bar{s}(g)=\bar{s}(f g)$ for all $f, g \in H^{0}$. To see that $\bar{s}(1)=1$, observe that by the foregoing $\bar{s}(1)$ is an idempotent which, by Lemma 1.4, is invertible.

(3) If $H$ is finite dimensional, then $H^{0}=H^{*}$ and the maps $i$ and $\Phi$ are bijective. Thus the arguments of (1) and (2) are reversible in this case. Finally, we must show that outer coactions of $H$ correspond to outer actions of $H^{*}$ if $\operatorname{dim}_{k} H<\infty$. This follows from the facts that if $\pi: H \rightarrow \bar{H}$ is a Hopf epimorphism, then ${ }^{t} \pi:(\bar{H})^{*} \rightarrow$ $H^{*}$ is a Hopf monomorphism and conversely, and that $A \otimes \bar{H}$ is isomorphic to $\operatorname{Hom}_{k}\left((\bar{H})^{*}, A\right)$ via the analogue of $i$, in finite dimensions. Thus, if $\rho$ is a weak coaction of $H$ on $A$ with associated weak action of $H^{*}$, then the induced weak coaction $\rho$ of $\bar{H}$ of $A$ is inner if and only if the restriction of the weak action of $H^{*}$ to $(\bar{H})^{*}$ inner.

If $H$ is not finite dimensional, the relationship between outer coactions of $H$ and outer actions of $H^{0}$ is much more complex: neither property implies the other. Before giving our examples, we need the following

LEMMA 2.7. For any field $k$, there exists a simple group $G$ such that $(k G)^{0}$ is trivial.

ProOF. Let $K$ be a field of cardinality greater than that of $k$; if $k$ is finite, assume $K$ is infinite. Let $G=\operatorname{PSL}(2, K)$, a simple group, and set $H=k G$. We claim that $H^{0}$ is trivial. In fact, let $f \in H^{0}$ and write $\Delta f=\sum_{j}{ }_{1}^{n} f_{j} \otimes g_{j}$. Then for any $h \in H, h \rightarrow f=\sum_{j}{ }_{1}^{n}\left\langle g_{j}, h\right\rangle f_{j}$ so that $V=H \rightarrow f$ is a finite dimensional representation space for $H$ and hence for $G$. Let $\pi$ denote this representation of $G$ on $V$. Since $G$ is simple, either $\pi$ is faithful or else $\pi=$ id. If $\pi$ were faithful it would give us an injection of $G$ into $\mathrm{GL}_{k}(V)$, contradicting our cardinality assumption. Hence $x \rightarrow f=(\pi x) f=f$ for all $x \in G$ and we have $h \rightarrow f=\varepsilon(h) f$ for $h \in H$, and so

$$
\langle f, h\rangle=\langle h \rightarrow f, 1\rangle=\varepsilon(h)\langle f, 1\rangle=\langle\langle f, 1\rangle \varepsilon, h\rangle \quad \text { for all } h \in H .
$$

Therefore $f=\langle f, 1\rangle \varepsilon$ for all $f \in H^{0}$, that is, $\operatorname{dim}_{k} H^{0}=1$.

EXAMPLE 2.8. Let $G$ be as in Lemma 2.7, set $A=H=k G$. Then the natural coaction of $H$ on $A$ is outer by Example 2.4, but $H^{0}$ is trivial and hence the corresponding action of $H^{0}$ is inner and not outer.

EXAMPLE 2.9. We produce a Hopf algebra $H$, an algebra $A$, and a coaction of $H$ on $A$ such that the coaction is not outer, but the corresponding $H^{0}$-action is outer. Let $1 \rightarrow N \stackrel{\alpha}{\rightarrow} F \stackrel{\pi}{\rightarrow} G \rightarrow 1$ be an exact sequence of groups with $F$ free and $G$ as in Lemma 2.7. Let $H=k F$ and $A=k N$. Our coaction $\rho$ is given by

$$
\rho: k N \stackrel{\Delta}{\rightarrow} k N \otimes k N \stackrel{\mathrm{id} \otimes \alpha}{\rightarrow} k N \otimes k F .
$$


This coaction is not outer: let $\bar{H}=k G$. Then $\pi: k F \rightarrow k G \rightarrow 0$ and the corresponding

$$
\bar{\rho}=(\mathrm{id} \otimes \pi) \circ(\mathrm{id} \otimes \alpha) \circ \Delta=(\mathrm{id} \otimes \varepsilon) \circ \Delta=\mathrm{id} \otimes \overline{1},
$$

the trivial coaction, which is certainly inner.

However we claim that the corresponding action of $H^{0}$ is outer. In fact, $H^{0}$ is dense in $H^{*}$ since $k F$ is residually finite dimensional (any free group is residually finite [15, p. 414]). Thus $H^{0}$ is not trivial. Let $L$ be a Hopf subalgebra of $H^{0}$ such that the induced action of $L$ on $A$ is inner. The argument of the second paragraph of Theorem 1.22 (with $A$ trivial and $H$ replaced by $k N$ ) shows that $\langle f, h\rangle=\varepsilon(h)\langle f, 1\rangle$ for all $f \in L, h \in k N$, that is, $f(x)=f(1)$ for all $x \in N$. In particular, $f\left(\omega_{N}\right)=0$, where $\omega_{N}=(\operatorname{Ker} \varepsilon) \cap k N$. But then $f\left(\omega_{N} \cdot k F\right)=0$, since $\omega_{N} \cdot k F$ is spanned by all $(1-n) x$ for $n \in N, x \in F$, and $f((1-n) x)=\langle\Delta f,(1-n) \otimes x\rangle$, and $\Delta f \in L \otimes L$.

Now $\operatorname{Ker}(\pi: k F \rightarrow k G)=\omega_{N} \cdot k F$ and so each $f \in L$ is of the form $f=\bar{f} \circ \pi$. Clearly, $\bar{f} \in(k G)^{0}$. By Lemma $2.7,(k G)^{0}$ is trivial. Thus $\bar{f}(y)=\bar{f}(1)$ for all $y \in G$, which implies $f(x)=f(1)$ for all $x \in F$. Therefore $f=\langle f, 1\rangle \varepsilon$ for all $f \in L$ and so $L$ is the trivial Hopf algebra.

This last example pinpoints exactly the obstruction to proving $H^{0}$-outer implies $H$-outer: $H$ has a nontrivial homomorphic image $\bar{H}$ with $(\bar{H})^{0}$ trivial. Indeed, we have

LEMMA 2.10. Let $H$ be a Hopf algebra such that whenever $\bar{H}$ is a Hopf homomorphic image of $H$ with $(\bar{H})^{0}$ trivial then $\bar{H}$ is trivial. Let $\rho$ be a weak coaction of $H$ on $A$ such that the corresponding action of $H^{0}$ is outer. Then $\rho$ is outer.

Proof. Let $\pi: H \rightarrow \bar{H}$ be a Hopf algebra epimorphism and suppose $\bar{\rho}=$ (id $\otimes \pi) \circ \rho$ is inner. As in the proof of Proposition 2.6(3), $\pi^{0}:(\bar{H})^{0} \rightarrow H^{0}$, where $\pi^{0}=\left.{ }^{t} \pi\right|_{(\bar{H})^{0}}$, is a Hopf algebra monomorphism, and the weak action of $(\bar{H})^{0}$ corresponding to $\rho$ is just the pullback via $\pi^{0}$ of the weak action of $H^{0}$ corresponding to $\rho$. By Proposition 2.6(1), the weak action of $(\bar{H})^{0}$ is inner and so $(\bar{H})^{0}$ is trivial. By hypothesis, so is $\bar{H}$.

A good class of Hopf algebras to which the lemma applies consists of all $H$ such that every Hopf homomorphic image is residually finite dimensional. If $H=k G$, this means that every homomorphic image of $G$ should be residually linear over $k$. Moreover all $U(L), L$ a finite dimensional Lie algebra over $k$, belong to this class, if $k$ has characteristic zero $[\mathbf{2 3}, \mathbf{7}]$.

The following remark and example show that finding reasonable conditions on infinite dimensional $H$ such that $H^{0}$-inner implies $H$ inner is a pretty hopeless task.

REMARK 2.11. Let $\rho: A \rightarrow A \otimes H$ be an inner weak coaction implemented by $s \in$ $A \otimes H$. Writing $s=\sum_{i} b_{i} \otimes h_{i}$ and $s^{-1}=\sum_{j} c_{j} \otimes l_{j}$, we have $\rho a=\sum_{i j} b_{i} a c_{j} \otimes h_{i} l_{j}$. Let $V$ be the linear span of $\left\{h_{i} l_{j}\right\}$ in $H$. Then $\rho A \subseteq A \otimes V$, where $\operatorname{dim}_{k} V<\infty$.

EXAMPLE 2.12. Let $H$ be any cocommutative Hopf algebra. Then $H$ is an $H^{0}$-module algebra and we may form $A=H \# H^{0}$. Define $\rho: A \rightarrow A \otimes H$ by

$$
\rho(h \# f)=\sum_{(h)}\left(h_{(1)} \# f\right) \otimes h_{(2)} .
$$

A simple calculation shows that $\rho$ is a $H$-coaction on $A$ : properties (2)-(4) of Definition 2.1 are immediate, and the verification of (1) follows from [2, Lemma 
1.1(i)] using cocommutativity. The corresponding $H^{0}$-action is given by

$$
g \cdot(h \# f)=\sum_{(h)}\left\langle g, h_{(2)}\right\rangle h_{(1)} \# f=(g \rightarrow h) \# f,
$$

and this is just the strongly inner action of $H^{0}$ on $H \# H^{0}$ implemented by $u \in \operatorname{Hom}_{k}\left(H^{0}, H \# H^{0}\right)$ defined by $u(g)=1 \# g$ as in Example 1.9 (note that the adjoint action of $H^{0}$ on itself is trivial since $H^{0}$ is commutative). Now suppose $\operatorname{dim}_{k} H=\infty$. We then claim $\rho$ is not inner. If $\rho$ were inner, then we would have $\rho A \subseteq A \otimes V$ for some finite dimensional $V$. Set $f=1$ in (2.13) and apply $\left(\varepsilon_{H} \otimes \varepsilon_{H^{0}}\right) \otimes$ id to both sides. We get

$$
\begin{aligned}
h & =\sum_{(h)} \varepsilon_{H}\left(h_{(1)}\right) \varepsilon_{H^{0}}(1) h_{(2)}=\left(\left(\varepsilon_{H} \otimes \varepsilon_{H^{0}}\right) \otimes \mathrm{id}\right) \rho(h \# 1) \\
& \in\left(\varepsilon_{H} \otimes \varepsilon_{H^{0}}\right)(A) V \subseteq V
\end{aligned}
$$

for all $h \in H$, a contradiction.

The difficulties appearing in Examples 2.8, 2.9, and 2.12 suggest that the definition of inner and outer coactions are defective and should be changed. When $H$ is residually finite dimensional, there is a reasonable alternative. For in that case $\operatorname{Hom}_{k}\left(H^{0}, A\right)$ is a completion of $A \otimes H$ via the injection $i$, and so it is natural to call a weak coaction $\rho: A \rightarrow A \otimes H$ "inner" if it becomes inner in $\operatorname{Hom}_{k}\left(H^{0}, A\right)$, that is, if there exists invertible $u \in \operatorname{Hom}_{k}\left(H^{0}, A\right)$ such that $i(\rho(a))=u * i(a \otimes 1) * u^{-1}$. Now $i(a \otimes 1)=\hat{a}$ as in (1.10). In view of (2.5) and (1.11), $\rho$ "inner" merely says that the weak $H^{0}$-action corresponding to $\rho$ is inner.

The process of completing $A \otimes H$ to $\operatorname{Hom}_{k}\left(H^{0}, A\right)$ via $i$ is reminiscent of the method used in $C^{*}$-algebras $[\mathbf{2 2}]$ and in $W^{*}$-algebras $[\mathbf{2 1}]$. Note that the unitary coactions of $[\mathbf{1 0}]$ are in fact strongly "inner", where "inner" here means that it is obtained by conjugating the trivial coaction in the authors' closure of $A \otimes H$.

Returning to our situation with $H$ residually finite dimensional, our examples suggest that $\rho$ should be called "outer" if for any residually finite dimensional Hopf homomorphic image $\bar{H}$ of $H, \bar{\rho}$ "inner" implies $\bar{H}$ trivial. For with this definition we only consider $\bar{H}$ such that $\operatorname{Hom}_{k}\left((\bar{H})^{0}, A\right)$ is a completion of $A \otimes \bar{H}$.

As we will see at the end of the next section, these notions of "inner" and "outer" may be more useful for gradings than the notions of Definition 2.2, which turn out to be extremely restrictive.

3. Two applications: graded algebras; semicenters of Hopf algebras. A major application of the notions in $\S 2$ is found in the theory of graded algebras.

Let $A$ be a $k$-algebra. Recall that $A$ is graded by the group $G$ if $A=\sum_{x \in G} \oplus A_{x}$, where each $A_{x}$ is a $k$-subspace of $A$, and if $A_{x} A_{y} \subseteq A_{x y}$ for all $x, y \in G$. It is not difficult to see that the $G$-gradings of $A$ are in bijective correspondence with the coactions of $k G$ on $A$ : the coaction $\rho: A \rightarrow A \otimes_{k} k G$ corresponding to a given grading of $A$ is given by $\rho a=\sum_{x \in G} a_{x} \otimes x$, where $a=\sum_{x \in G} a_{x}$ with $a_{x} \in A_{x}$ for $x \in G[2$, Lemma 4.8].

As in $\S 2$, it follows that a $G$-graded algebra $A$ is a $(k G)^{0}$-module algebra. The converse is false. If $k G$ is residually finite dimensional (i.e., $G$ is residually linear $[\mathbf{2 3}])$, then a $(k G)^{0}$-module algebra $A$ is graded in such a way that the coaction $\rho$ of $k G$ gives rise to the original $(k G)^{0}$-action if and only if that action is $k G$-locally 
finite (see [2, Corollary 4.9]). If $G$ is finite, the $(k G)^{*}$-action is given in terms of the grading by

$$
f \cdot a=\sum_{x \in G}\langle f, x\rangle a_{x}
$$

for $a \in A, f \in(k G)^{*}$.

In the special case when $G$ is finite abelian of exponent $n$ and $k$ contains a primitive $n$th root of 1 , then $(k G)^{*}$ is Hopf isomorphic to $k \hat{G}$, where $\hat{G}=\operatorname{Hom}\left(G, k^{\times}\right)$ is the dual group. Then a $G$-grading of $A$ coincides with a $\hat{G}$ action on $A$ : if $\lambda \in \hat{G} \subseteq(k G)^{*}$, then $\lambda \cdot a$ is given by (3.1). Conversely, if $\hat{G}$ acts on $A$, then the $G$-grading is given by

$$
A_{x}=\{a \in A: \lambda \cdot a=\langle\lambda, x\rangle a \text { for all } \lambda \in \hat{G}\} .
$$

We now wish to consider inner gradings.

DEFINITION 3.3. A $G$-grading of $A$ is inner (resp. strongly inner, resp. outer) if the corresponding $k G$-coaction is inner (resp. strongly inner, resp. outer) in the sense of Definition 2.2.

Let us clarify the notion of outer grading. If $H=k G$ and if $\bar{H}$ is a Hopf homomorphic image of $H$, then $\bar{H}=k \bar{G}$, where $\bar{G}$ is a homomorphic image of the group $G$. In fact, if $\pi: H \rightarrow \bar{H}$ is a Hopf epimorphism, then $\pi$ maps $G$ into the set of group-like elements of $\bar{H}$ and is a group homomorphism. Let $\bar{G}=\pi G$. Clearly, $\bar{H}$ is spanned by $\bar{G}$, and since distinct group-like elements in a Hopf algebra are linearly independent $[\mathbf{2 5}$, p. 56] we have $\bar{H}=k \bar{G}$. The converse of this statement is obvious. Next we observe that if $A$ is $G$-graded and $\bar{G}$ is a homomorphic image of $G$, then the $\bar{G}$-grading of $A$ as in Definiton 2.2(3) is gotten as follows: if $\bar{x} \in \bar{G}$, then $A_{\bar{x}}=\sum_{x \in \bar{x}} \oplus A_{x}$. In fact,

$$
\bar{\rho} a=(\mathrm{id} \otimes \pi) \rho a=\sum_{x \in G} a_{x} \otimes \bar{x}=\sum_{\bar{x} \in \bar{G}}\left(\sum_{x \in \bar{x}} a_{x}\right) \otimes \bar{x} .
$$

Consequently, we have

CoRollary 3.4. A G-grading of $A$ is outer if and only if $G \neq\{1\}$ and, whenever the $\bar{G}$-grading of $A$ given by $A_{\bar{x}}=\sum_{x \in \bar{x}} \oplus A_{x}, \bar{x} \in \bar{G}, \bar{G}$ a homomorphic image of $G$, is inner, then $\bar{G}=\{1\}$.

We next consider strongly inner gradings.

Proposition 3.5. Let $A$ be strongly inner $G$-graded. Then there exists a finite subset $T \subset G$ and an injective map $x \mapsto e_{x}$ of $T$ onto an orthogonal set of nonzero idempotents of $A$ such that $\sum_{x \in T} e_{x}=1$ and such that

$$
a_{x}=\sum_{y \in T \cap x T} e_{y} a e_{x^{-1} y}
$$

for all $x \in G, a \in A$. In particular $A_{x}=\{0\}$ if $T \cap x T=\varnothing$, so that $A_{x} \neq\{0\}$ implies that $x \in T T^{-1}$.

Conversely, let $T$ be any finite subset of $G$ and suppose we are given an injective map $x \mapsto e_{x}$ of $T$ onto an orthogonal set of nonzero idempotents of $A$ such that $\sum_{x \in T} e_{x}=1$. Then (3.6) defines a strongly inner $G$-grading of $A$. 
Proof. From Definition 2.2(2), there exists an invertible $s \in A \otimes k G$ such that $\rho a=s(a \otimes 1) s^{-1}$ for all $a \in A$ and such that $\mu(s \otimes s)=($ id $\otimes \Delta) s$. Expanding $s$ with respect to the basis $G$ of $k G$, we write $s=\sum_{x \in T} e_{x} \otimes x$, where $T$ is a finite subset of $G$ and $e_{x} \neq 0$ for $x \in T$. We have

$$
\begin{aligned}
\sum_{x, y \in T} e_{x} e_{y} \otimes x \otimes y & =\mu(s \otimes s)=(\mathrm{id} \otimes \Delta) s \\
& =\sum_{x \in T} e_{x} \otimes x \otimes x .
\end{aligned}
$$

It follows that $e_{x} e_{y}=\delta_{x y} e_{x}$ for $x, y \in T$ so that $\left\{e_{x}: x \in T\right\}$ is an orthogonal set of nonzero idempotents. Let $e=\sum_{x \in T} e_{x}$. Then $(e \otimes 1) s=s$. Since $s$ is invertible, so is $e \otimes 1$ and hence so is $e$. Thus $e=1$. Then direct calculation shows $s^{-1}=\sum_{x \in T} e_{x} \otimes x^{-1}$. Finally

$$
\begin{aligned}
\sum_{z \in G} a_{z} \otimes z & =s(a \otimes 1) s^{-1}=\sum_{x, y \in T} e_{x} a e_{y} \otimes x y^{-1} \\
& =\sum_{z \in G}\left(\sum_{\substack{x \in T \\
z^{-1} x \in T}} e_{x} a e_{z^{-1} x}\right) \otimes z
\end{aligned}
$$

verifying (3.6).

Conversely, if the $e_{x}, x \in T$ are given, set

$$
A_{x}=\sum_{\substack{y z^{-1}=x \\ y, z \in T}} e_{y} A e_{z} \quad \text { for all } x \in G .
$$

It is then easy to check that the $\left\{A_{x}: x \in G\right\}$ form a $G$-grading of $A$ and that the corresponding maps $a \mapsto a_{x}, x \in G$, are given by (3.6). Finally, setting $s=$ $\sum_{x \in T} e_{x} \otimes x$, we see that $s^{-1}=\sum_{x \in T} e_{x} \otimes x^{-1}$, that $\sum_{x \in G} a_{x} \otimes x=s(a \otimes 1) s^{-1}$, and that $\mu(s \otimes s)=($ id $\otimes \Delta) s$, all by direct calculation.

When $G$ is finite, Proposition 3.5 can be proved by noting that a $k G$-coaction is just a $(k G)^{*}$-action, and that as an algebra $(k G)^{*}$ is just a direct sum of $|G|$ copies of $k:(k G)^{*}=\bigoplus_{x \in G} k p_{x}$, where $\left\{p_{x}: x \in G\right\}$ is the basis dual to $\{x: x \in G\}$. Thus the $\left\{p_{x}: x \in G\right\}$ are an orthogonal set of idempotents in $(k G)^{*}$ and $\sum_{x \in G} p_{x}=1$. If $u \in \operatorname{Alg}\left((k G)^{*}, A\right)$, we may set $e_{x}=u\left(p_{x}\right), x \in G$, and obtain an orthogonal set of idempotents in $A$ summing to 1 . By Lemma 1.16, the strongly inner action of $(k G)^{*}$ implemented by $u$ just gives $p_{x} \cdot a=\sum_{y \in G} e_{y} a e_{x^{-1} y}$, which is (3.6). Conversely, any map $x \mapsto e_{x}$, from $G$ to an orthogonal set of idempotents in $A$ summing to 1 gives rise to $u \in \operatorname{Alg}\left((k G)^{*}, A\right)$ such that $e_{x}=u\left(p_{x}\right), x \in G$.

Turning to examples, we note that any matrix ring $A=M_{n}(R)$ can be strongly inner graded by any group $G$ such that $|G| \geq n$ : just apply Proposition 3.5 to any bijection between a subset $T \subseteq G$ with $|T|=n$ and the matrix units $\left\{e_{i i}: i=\right.$ $1, \ldots, n\}$ of $M_{n}(R)$.

Other examples of our concepts can be obtained from some examples from $\S \S 1$ and 2 above.

First, consider the group algebra $A=k G$. $A$ is naturally $G$-graded: let $A_{x}=k x$, $x \in G$. As an $H=k G$-coaction this is just an instance of Example 2.4: $\rho=\Delta$. Hence the $G$-grading of $k G$ is outer if $G \neq\{1\}$. 
Next, consider a twisted group algebra $A=k_{\tau}[G]$, where $\tau: G \times G \rightarrow k$ is a normalized 2-cocycle. Recall that $A$ has a $k$-basis $\{\bar{x}: x \in G\}$ and that the multiplication in $A$ is given by $\bar{x} \bar{y}=\tau(x, y) \overline{x y}$. Again, $A$ is naturally $G$-graded by letting $A_{x}=k \bar{x}, x \in G$. Unlike the previous case (where $\tau \equiv 1$ ), this grading need not be outer, as we now show:

EXAMPLE 3.6. Let $A=C_{2}(k), G=\mathbf{Z}_{2} \times \mathbf{Z}_{2}$, as in Example 1.18, whose notation we use here. Settin $\overline{1}=1, \bar{x}=e_{1}, \bar{y}=e_{2}$, and $\overline{x y}=e_{1} e_{2}$ we see that $A=k_{\tau}[G]$ for suitable $\tau$. Thus $A$ is $G$-graded. We compute the corresponding $\hat{G}$-action and compare it with the $G$-action described in the earlier example. Let $w \mapsto \lambda_{w}$ be the isomorphism of $G$ onto $\hat{G}$ given by $\lambda_{x}(x)=\lambda_{y}(y)=-1$ and $\lambda_{x}(y)=\lambda_{y}(x)=1$. Then the definition of the action of $G$ in Example 1.18 comes to $x \cdot \bar{z}=\lambda_{x}(z) \bar{z}$ and $y \cdot \bar{z}=\lambda_{y}(z) \bar{z}$ for $z \in G$, so that $w \cdot \bar{z}=\lambda_{w}(z) \bar{z}$ for $z, w \in G$. But (3.1) says that $\lambda \cdot \bar{z}=\lambda(z) \bar{z}$ for $\lambda \in \hat{G}, z \in G$. We conclude that $w \cdot \bar{z}=\lambda_{w} \cdot \bar{z}$ for all $z, w \in G$, that is, $w$ and $\lambda_{w}$ have the same action on $A$ for all $w \in G$. Now the action of $G$ on $A$ was shown to be inner, but not strongly inner. Therefore the natural $G$-grading of $k_{\tau}[G]$ is inner, but not strongly inner. It is certainly not outer.

We next consider the relation between $G$-gradings for $G$ infinite and the corresponding $(k G)^{0}$-actions. Examples 2.8 and 2.9 are examples of $k G$-coactions and hence of $G$-gradings, and Example 2.12 becomes a graded ring example by choosing $H=k G, G$ any infinite group whatsoever. Thus it is possible for a $G$-grading to be outer but the action of $(k G)^{0}$ to be inner, for the $(k G)^{0}$-action to be outer but not the $G$-grading, or for the $(k G)^{0}$-action to be inner but not the $G$-grading. On the positive side, we can combine Lemma 2.10 (and the remarks following it) with Corollary 3.4 to get

COROLlaRY 3.7. Let $G$ be a group such that every homomorphic image of $G$ is residually $k$-linear. Let $A$ be a $G$-graded algebra such that the corresponding $(k G)^{0}$-action is outer. Then the $G$-grading is outer.

Finally we note that for any inner $G$-gradings, only a finite number of components $A_{x}$ can be nonzero according to Remark 2.11. This is very restrictive indeed. Intuitively the grading of Example 2.12 with $H=k G$ ought always to be an inner grading: for example, if $G=\mathbf{Z}$, then $\hat{G}=k^{\times}$, the multiplicative group of nonzero elements of $k$, and $\hat{G}$ acts on $A$ in an inner fashion. When $G$ is such that all homomorphic images are residually $k$-linear, the notions of "inner" and "outer" (see end of $\S 2$ ) are probably preferable.

We now turn to our second application.

When a Hopf algebra $H$ acts on an algebra $A$, one can form the subring $A^{H}$ of $H$-invariants, defined by

$$
A^{H}=\{a \in A: h \cdot a=\varepsilon(h) a, \text { all } h \in H\} .
$$

In the case of automorphisms and derivations, a larger subalgebra of $A$, called the "semi-invariant" subalgebra has been studied. For a group $G$ acting on $A$, an element $a \in A$ is a semi-invariant for $\lambda \in \hat{G}$ if $g \cdot a=\lambda(g) a$, for all $g \in G$; similarly if a Lie algebra $L$ acts on $A$, then $a$ is a semi-invariant for $\lambda \in L^{*}$ if $x \cdot a=\lambda(x) a$, for all $x \in L$. This idea has proved particularly interesting in the case of $L$ acting on $U(L)$ and its quotient division ring via the adjoint representation [6]. These 
notions extend to an arbitrary Hopf algebra and illustrate some of the concepts developed above.

Definition 3.8. Let $H$ act on $A$ and let $\lambda \in H^{*}$. Set

$$
A_{\lambda}=\{a \in A: h \cdot a=\lambda(h) a \text { for all } h \in H\} .
$$

$\bigcup_{\lambda \in H^{*}} A_{\lambda}$ is called the set of $H$-semi-invariants of $A$.

Note that $A_{\varepsilon}=A^{H}$, so that an invariant is a semi-invariant. Also compare with (3.2) above.

LEMMA 3.9. (1) If $A_{\lambda} \neq\{0\}$, then $\lambda \in \operatorname{Alg}(H, k)=G$, the group of grouplike elements of $H^{0}$.

(2) If $\lambda, \mu \in G$, then $A_{\lambda} A_{\mu} \subseteq A_{\lambda \mu}$.

(3) The sum $\sum_{\lambda \in G} A_{\lambda}$ is direct.

Proof. (1) Suppose $0 \neq a \in A_{\lambda}$ and let $h, l \in H$. Then $\lambda(h l) a=h l \cdot a=$ $h \cdot(l \cdot a)=\lambda(h) \lambda(l) a$ and $\lambda(1) a=1 \cdot a=a$. Thus $\lambda \in \operatorname{Alg}(H, k)=G$.

(2) Let $a \in A_{\lambda}, b \in A_{\mu}, h \in H$. Then

$$
h \cdot a b=\sum_{(h)}\left(h_{(1)} \cdot a\right)\left(h_{(2)} \cdot b\right)=\sum_{(h)} \lambda\left(h_{(1)}\right) \mu\left(h_{(2)}\right) a b=(\lambda \mu)(h) a b .
$$

(3) is well known.

DEFINITION 3.10. The semi-invariant subalgebra $S_{A}$ of $A$ is defined to be $\sum_{\lambda \in G} A_{\lambda}$.

Note that Lemma 3.9 assures us that $S_{A}$ is closed under multiplication. Moreover $1 \in A_{\varepsilon}$. Thus $S_{A}$ is a subalgebra of $A$. Also note that Lemma 3.9 says that $S_{A}$ is graded by $G$.

We now specialize to the case where $A=H$ and $H$ acts on $H$ by the adjoint action (Example 1.8). In this case $S_{H}$ is called the semicenter of $H$. Let $\lambda \in G$. Define $\phi_{\lambda}: H \mapsto H$ by $\phi_{\lambda}(h)=h-\lambda^{-1}=\sum_{(h)} \lambda^{-1}\left(h_{(1)}\right) h_{(2)}$.

LEMMA 3.11. The map $\lambda \mapsto \phi_{\lambda}$ is a homomorphism of $G$ into Aut $H$.

PrOOF. We have

$$
\begin{aligned}
\phi_{\lambda}(h l) & =\sum_{(h)(l)} \lambda^{-1}\left(h_{(1)} l_{(1)}\right) h_{(2)} l_{(2)}=\sum_{(h)(l)} \lambda^{-1}\left(h_{(1)}\right) \lambda^{-1}\left(l_{(1)}\right) h_{(2)} l_{(2)} \\
& =\phi_{\lambda}(h) \phi_{\lambda}(l),
\end{aligned}
$$

and $\phi_{\lambda}(1)=1 \leftarrow \lambda^{-1}=1$.

Finally,

$$
\phi_{\lambda \mu}(h)=h \leftarrow(\lambda \mu)^{-1}=\left(h-\mu^{-1}\right)-\lambda^{-1}=\phi_{\lambda}\left(\phi_{\mu}(h)\right)
$$

and

$$
\phi_{\varepsilon}(h)=\sum_{(h)} \varepsilon\left(h_{(1)}\right) h_{(2)}=h .
$$

PROPOSITION 3.12. Let $H$ act on itself via the adjoint action.

(1) $a \in H_{\lambda}, \lambda \in G$, if and only if $a h=\phi_{\lambda}(h) a$ for all $h \in H$.

(2) $\phi_{\mu}\left(H_{\lambda}\right)=H_{\mu \lambda \mu^{-1}}$.

(3) The center $Z(H)$ of $H$ equals $H_{\varepsilon}\left(=H^{H}\right)$. 
ProOF. (1) Let $a \in H_{\lambda}, h \in H$. Then by Lemma 1.14 and [2, Lemma 1.1], we have

$$
\begin{aligned}
\phi_{\lambda}(h) a & =\left(h-\lambda^{-1}\right) a=\sum_{(h)}\left[\left(h_{(1)}-\lambda^{-1}\right) \cdot a\right] h_{(2)}=\sum_{(h)} \lambda\left(h_{(1)}-\lambda^{-1}\right) a h_{(2)} \\
& =\sum_{(h)} \lambda^{-1}\left(h_{(1)}\right) \lambda\left(h_{(2)}\right) a h_{(3)}=\sum_{(h)} \varepsilon\left(h_{(1)}\right) a h_{(2)}=a h .
\end{aligned}
$$

Conversely, suppose $a h=\phi_{\lambda}(h) a$ for all $h \in H$. Then

$$
\begin{aligned}
h \cdot a & =\sum_{(h)} h_{(1)} a\left(S h_{(2)}\right)=\sum_{(h)} h_{(1)} \phi_{\lambda}\left(S h_{(2)}\right) a=\sum_{(h)} h_{(1)}\left(S h_{(2)}-\lambda^{-1}\right) a \\
& =\sum_{(h)} \lambda^{-1}\left(S h_{(3)}\right) h_{(1)}\left(S h_{(2)}\right) a=\lambda^{-1}(S h) a=\lambda(h) a \quad \text { for all } h \in H .
\end{aligned}
$$

(2) Let $a \in H_{\lambda}, h \in H$. Then by (1) we have $a \phi_{\mu^{-1}}(h)=\phi_{\lambda \mu^{-1}}(h) a$. Applying $\phi_{\mu}$ to both sides gives $\phi_{\mu}(a) h=\phi_{\mu \lambda \mu^{-1}}(h) \phi_{\mu}(a)$. Thus $\phi_{\mu}\left(H_{\lambda}\right) \subseteq H_{\mu \lambda \mu^{-1}}$. Therefore $\phi_{\mu^{-1}}\left(H_{\mu \lambda \mu^{-1}}\right) \subseteq H_{\lambda}$ so that $H_{\mu \lambda \mu^{-1}} \subseteq \phi_{\mu}\left(H_{\lambda}\right)$.

(3) $a \in H_{\varepsilon}$ if and only if $a h=\phi_{\varepsilon}(h) a=h a$ for all $h \in H$ by (1).

We remark that Proposition 3.12 shows that the semicenter $S_{H}$ of $H$ contains the center and not only is graded by $G$ but also is acted upon by $G$. Also Proposition $3.12(3)$ answers a question of Nichols [8, p. 45].

When $H=U(L)$, it is known that the semicenter of $H$ is commutative [6, Proposition 4.3.5]. Although false in general for $H=k G$, it is true whenever $k G$ is prime. For in that case, the torsion f.c. subgroup $\Delta^{+}(G)=1$, and so the f.c. subgroup $\Delta(G)$ is torsion free abelian. If $a \in(k G)_{\lambda}$, then $(\operatorname{ad} x) a=x a x^{-1}=\lambda(x) a$ for all $x \in G$ implies that $a \in k \Delta$; thus any two such semi-invariants commute. Thus we ask: is it always true that the semicenter of a prime cocommutative Hopf algebra is commutative?

If $k$ is algebraically closed of characteristic 0 , recent work of Montgomery and Passman [20] shows that the answer is "yes". That argument used the fact that in this case, $H=U(L) * G$. In this section we show that any two semi-invariants "almost" commute - that is, they commute up to a central element in a localization of $H$.

LEMMA 3.13. Let $H$ be a prime Hopf algebra. Then the set $S$ of nonzero semi-invariants is a (left) Ore set for $H$.

ProOF. First, the product of two semi-invariants is a semi-invariant by Lemma 3.9. Second, $S$ consists of non-zero-divisors. For if $s \in H_{\lambda}, s \neq 0$, but $x s=0$, then $0=x s H=x \phi_{\lambda}(H) s=x H s$. Since $H$ is prime, $x=0$. Similarly $s x=0$ implies $x=0$. Thus $S$ is a multiplicatively closed set of regular elements. Moreover, given any $s \in S$ and $h \in H, s h=\phi_{\lambda}(h) s$ for some $\lambda \in G$; thus the (left) Ore condition is satisfied.

We may thus form the localization $H_{S}=\left\{s^{-1} h \mid h \in H, s \in S\right\}$.

THEOREM 3.14. Let $H$ be any prime Hopf algebra.

(1) The center $Z\left(H_{S}\right)=\left\{s_{1}^{-1} s_{2}\right.$ : there exists $\lambda$ with $\left.s_{1}, s_{2} \in H_{\lambda}, s_{1} \neq 0\right\}$.

(2) If $s_{1}, s_{2} \in H_{\lambda}$, any $\lambda$, then $s_{1} s_{2}=s_{2} s_{1}$.

(3) If $H$ is cocommutative and $s \in H_{\lambda}, t \in H_{\mu}$, then st $=$ zts, where $z \in Z\left(H_{S}\right)$. 
PROOF. (1) Note that in $H_{S}, \phi_{\lambda}(h)=s_{1} h s_{1}^{-1}=s_{2} h s_{2}^{-1}$, and thus $s_{1}^{-1} s_{2}$ is central. Conversely, say that $z=s^{-1} h \in Z\left(H_{S}\right)$, where $s \in H_{\lambda}$. Then for all $l \in H, s^{-1} h l=l s^{-1} h$. That is, $h l=s l s^{-1} h=\phi_{\lambda}(l) h$, for all $l \in H$. Now by part (1) of Proposition 3.12, $h \in H_{\lambda}$ also.

(2) If $0 \neq s_{1}, s_{2} \in H_{\lambda}$, then by (1) $s_{1}^{-1} s_{2} \in Z\left(H_{S}\right)$. Thus $\left(s_{1}^{-1} s_{2}\right) s_{2}=s_{2}\left(s_{1}^{-1} s_{2}\right)$. Since $s_{2}$ is invertible in $H_{S}$, this gives $s_{1}^{-1} s_{2}=s_{2} s_{1}^{-1}$. But then $s_{2} s_{1}=s_{1} s_{2}$.

(3) Since $H$ is cocommutative, $\lambda \mu=\mu \lambda$. Thus both $s t$ and $t s$ are in $H_{\lambda \mu}$. Then by $(2),(s t)(t s)=(t s)(s t)$, and so $s t=\left[(t s)^{-1}(s t)\right] t s=z t s$, since $z=(t s)^{-1} s t \in$ $Z\left(H_{S}\right)$ by $(1)$. The theorem is proved.

Notice that the above arguments show that each semi-invariant of $H$ determines an automorphism of $H$ which becomes inner on $H_{S}$; such automorphisms are called " $X$-inner" [16] (although the usual definition of $X$-inner uses the Martindale quotient ring $Q_{0}(H)$ of $H$, it is easy to see that $H_{S} \subseteq Q_{0}(H)$ ). In the case $H=U(L)$, it is known that the only $X$-inner automorphisms of $H$ are induced by semi-invariants of $Q_{0}(H)[\mathbf{1 7}]$; however for $H=k G$, and more generally for $H=U(L) \# k G$, other $X$-inner automorphisms are possible $[\mathbf{1 9}, \mathbf{2 0}]$.

We note that part (1) of Theorem 3.14 cannot be improved to elements of the form $z_{1}^{-1} z_{2}$, for $z_{1}, z_{2} \in Z(H)$. For, this is false for $H=U(L)[6, \S 4.9 .8]$.

4. Crossed products. We now turn our attention to crossed products, that is, smash products where the multiplication is twisted by a cocycle $\sigma$. As mentioned in the Introduction, we want to consider the very general situation where $H$ is an arbitrary Hopf algebra acting weakly on an algebra $A$ and where $\sigma$ is some sort of cocycle from $H \times H$ to $A$. The correct setting for this sort of construction is the following:

DEFINITION 4.1. Let $H$ be a Hopf algebra with a weak action on the algebra $A$, and let $\sigma: H \times H \rightarrow A$ be a $k$-bilinear map. Let $A \#_{\sigma} H$ be the (in general nonassociative) algebra (in general without 1 ) whose underlying vector space is $A \otimes_{k} H$ and whose multiplication is given by

$$
(a \otimes h)(b \otimes l)=\sum_{(h),(l)} a\left(h_{(1)} \cdot b\right) \sigma\left(h_{(2)}, l_{(1)}\right) \otimes h_{(3)} l_{(2)}
$$

for all $a, b \in A, h, l \in H$. The element $a \otimes h$ of $A \#_{\sigma} H$ will usually be written $a \# h$ to remind us that $H$ is (weakly) acting on $A$. The algebra $A \#_{\sigma} H$ is called a crossed product if it is associative with 1 \# 1 as identity element.

We now determine simple necessary and sufficient conditions on $\sigma$ and the weak action for $A \#_{\sigma} H$ to be a crossed product.

DEFINITION 4.3. A bilinear map $\sigma: H \times H \rightarrow A$ is called normal if $\sigma(1, h)=$ $\sigma(h, 1)=\varepsilon(h) 1$ for all $h \in H$.

Lemma 4.4. $1 \# 1$ is the identity element of $A \#_{\sigma} H$ if and only if $\sigma$ is normal.

PROOF.

$$
(1 \# 1)(a \# h)=\sum_{(h)}(1 \cdot a) \sigma\left(1, h_{(1)}\right) \# h_{(2)}=\sum_{(h)} a \sigma\left(1, h_{(1)}\right) \# h_{(2)}
$$


by Definitions 4.2 and 1.1(3). Thus $\sigma(1, h)=\varepsilon(h) 1$, all $h \in H$ implies $1 \# 1$ is a left identity. Conversely, suppose $1 \# 1$ is a left identity. Setting $a=1$ and applying id $\otimes \varepsilon$ to both sides, we get

$$
\varepsilon(h) 1 \otimes 1=\sum_{(h)} \sigma\left(1, h_{(1)}\right) \otimes \varepsilon\left(h_{(1)}\right) 1=\sigma(1, h) \otimes 1 .
$$

Similarly, 1 \# 1 is a right identity if and only if $\sigma(h, 1)=\varepsilon(h) 1$, all $h \in H$.

Lemma 4.5. Assume that $\sigma(h, 1)=\varepsilon(h) 1$, all $h \in H$. Then $A \#_{\sigma} H$ is associative if and only if the following two conditions hold:

(1) (cocycle condition) For all $h, l, m \in H$ we have

$$
\sum_{(h)(l)(m)}\left[h_{(1)} \cdot \sigma\left(l_{(1)}, m_{(1)}\right)\right] \sigma\left(h_{(2)}, l_{(2)} m_{(2)}\right)=\sum_{(h)(l)} \sigma\left(h_{(1)}, l_{(1)}\right) \sigma\left(h_{(2)} l_{(2)}, m\right) .
$$

(2) (twisted module condition) For all $h, l \in H, a \in A$ we have

$$
\sum_{(h)(l)}\left(h_{(1)} \cdot\left(l_{(1)} \cdot a\right)\right) \sigma\left(h_{(2)}, l_{(2)}\right)=\sum_{(h)(l)} \sigma\left(h_{(1)}, l_{(1)}\right)\left(h_{(2)} l_{(2)} \cdot a\right) .
$$

(This is our generalization of (0.1).)

Proof. Suppose $A \#_{\sigma} H$ is associative. Then (1) and (2) follow from

$$
(1 \# h)[(1 \# l)(1 \# m)]=[(1 \# h)(1 \# l)](1 \# m)
$$

and

$$
(1 \# h)[(1 \# l)(a \# 1)]=[(1 \# h)(1 \# l)](a \# 1),
$$

respectively, by applying id $\otimes \varepsilon$ to both sides (and using the hypothesis on $\sigma$ in the second case only). Conversely, suppose (1) and (2) hold. Let $a, b, c \in A$ and $h, l, m \in H$. Then

$$
\begin{aligned}
(a \# & h)[(b \# l)(c \# m)] \\
= & \sum_{(h)(l)(m)} a\left(h_{(1)} \cdot\left[b\left(l_{(1)} \cdot c\right) \sigma\left(l_{(2)}, m_{(1)}\right)\right]\right) \sigma\left(h_{(2)}, l_{(3)} m_{(2)}\right) \# h_{(3)} l_{(4)} m_{(3)} \\
= & \sum_{(h)(l)(m)} a\left(h_{(1)} \cdot b\right)\left(h_{(2)} \cdot\left(l_{(1)} \cdot c\right)\right)\left(h_{(3)} \cdot \sigma\left(l_{(2)}, m_{(1)}\right)\right) \sigma\left(h_{(4)}, l_{(3)} m_{(2)}\right) \# h_{(5)} l_{(4)} m_{(3)} \\
= & \sum_{(h)(l)(m)} a\left(h_{(1)} \cdot b\right)\left(h_{(2)} \cdot\left(l_{(1)} \cdot c\right)\right) \sigma\left(h_{(3)}, l_{(2)}\right) \sigma\left(h_{(4)} l_{(3)}, m_{(1)}\right) \# h_{(5)} l_{(4)} m_{(2)} \\
= & \sum_{(h)(l)(m)} a\left(h_{(1)} \cdot b\right) \sigma\left(h_{(2)}, l_{(1)}\right)\left(h_{(3)} l_{(2)} \cdot c\right) \sigma\left(h_{(4)} l_{(3)}, m_{(1)}\right) \# h_{(5)} l_{(4)} m_{(2)} \\
= & {[(a \# h)(b \# l)](c \# m) . }
\end{aligned}
$$

The first and fifth equalities come from (4.2), the second from (1.1(1)), and the third and fourth from (1) and (2) above, respectively.

COROLlary 4.6. A \# $\#_{\sigma} H$ is a crossed product if and only if $\sigma$ is a normal cocycle and the twisted module condition holds.

Let us return for a moment to Sweeder's situation in $[\mathbf{2 4}, \S 8]: H$ is cocommutative and $A$ is a commutative $H$-module algebra. Our twisted module condition is then automatically satisfied, and our cocycle condition is equivalent to that of [24, Lemma 8.1(a)]. We have the following partial converse. 
LEMMA 4.7. Let $\sigma$ and the weak action of $H$ on $A$ satisfy the twisted module condition. Suppose $H$ is cocommutative, $\sigma$ is invertible as a member of $\operatorname{Hom}_{k}(H \otimes H, A)$, and $\sigma(H \times H) \subseteq Z(A)$. Then $A$ is an $H$-module.

PROOF. Let $a \in A$ and define $\mu_{1}, \mu_{2} \in \operatorname{Hom}_{k}(H \otimes H, A)$ by $\mu_{1}(h, l)=h \cdot(l \cdot a)$ and $\mu_{2}(h, l)=h l \cdot a$. Then the twisted module condition is just $\mu_{1} * \sigma=\sigma * \mu_{2}$, which equals $\mu_{2} * \sigma$ since $H$ is cocommutative and $\sigma$ has central values. Since $\sigma$ is invertible, $\mu_{1}=\mu_{2}$.

Here is a partial converse in a different direction:

PROPOSITION 4.8. Let $A$ be an $H$-module algebra and suppose that $\sigma$ and this $H$-action satisfy the twisted module condition. If $H$ is cocommutative, then $\sigma(H \times H) \subseteq Z(A)$.

PROOF. It suffices to prove this when $k$ is algebraically closed. Then $H$ is pointed and we have $H=\bigoplus_{x \in G} H^{x}$, where $G$ is the group of group-like elements in $H$ and where $H^{x}$ is the irreducible component of $H$ containing $x$ as its unique group-like element [25, p. 174]. For each $x \in G$, set $H_{0}^{x}=k x$ and define inductively

$$
H_{n}^{x}=\left\{h \in H^{x}: \Delta h \in x \otimes H^{x}+H^{x} \otimes H_{n-1}^{x}\right\}
$$

for $n=1,2, \ldots$ Then $\left[\mathbf{2 5}\right.$, p. 185] we know that $H_{n+1}^{x} \supseteq H_{n}^{x}$ and that $H^{x}=\bigcup_{n} H_{n}^{x}$. Set $H_{-1}^{x}=\{0\}$. Now let $h \in H_{n}^{x}$. Then $\Delta h=x \otimes h^{\prime \prime \prime}+\sum_{i} h_{i}^{\prime} \otimes h_{i}^{\prime \prime}$ with $h^{\prime \prime \prime}, h_{i}^{\prime} \in H^{x}$ and $h_{i}^{\prime \prime} \in H_{n-1}^{x}$. Applying $\varepsilon \otimes$ id we get $h=h^{\prime \prime \prime}+\sum_{i} \varepsilon\left(h_{i}^{\prime}\right) h_{i}^{\prime \prime}$ so that $h^{\prime \prime \prime}=h$ $\left(\bmod H_{n-1}^{x}\right)$. We may therefore replace $h^{\prime \prime \prime}$ by $h$, absorbing the difference into the other terms. We have shown that $h \in H_{n}^{x}, n \geq 0$, implies

$$
\Delta h=x \otimes h+\sum_{i} h_{i}^{\prime} \otimes h_{i}^{\prime \prime}
$$

with $h_{i}^{\prime} \in H^{x}, h_{i}^{\prime \prime} \in H_{n-1}^{x}$. $H$ cocommutative gives also

$$
\Delta h=h \otimes x+\sum_{i} h_{i}^{\prime \prime} \otimes h_{i}^{\prime}
$$

with the same $h$ 's.

Now partially order $\mathbf{Z} \times \mathbf{Z}$ by $\left(n^{\prime}, m^{\prime}\right) \leq(n, m)$ if $n^{\prime} \leq n, m^{\prime} \leq m$. Let $x, y \in G$. Clearly $\sigma\left(H_{n}^{x}, H_{m}^{y}\right)=\{0\} \subseteq Z(A)$ if $(n, m) \nsupseteq(0,0)$. So assume $(n, m) \geq(0,0)$ and that $\sigma\left(H_{n^{\prime}}^{x}, H_{m^{\prime}}^{y}\right) \subseteq Z(A)$ for $\left(n^{\prime}, m^{\prime}\right)<(n, m)$. Let $h \in H_{n}^{x}, l \in H_{m}^{y}$ and write $\Delta h$ as above and also

$$
\Delta l=y \otimes l+\sum_{j} l_{j}^{\prime} \otimes l_{j}^{\prime \prime}=l \otimes y+\sum_{j} l_{j}^{\prime \prime} \otimes l_{j}^{\prime} .
$$

Substituting these expressions into $4.5(2)$ we get

$$
\begin{aligned}
(x y \cdot a) & \sigma(h, l)+\sum_{j}\left(x l_{j}^{\prime} \cdot a\right) \sigma\left(h, l_{j}^{\prime \prime}\right)+\sum_{i}\left(h_{i}^{\prime} y \cdot a\right) \sigma\left(h_{i}^{\prime \prime}, l\right) \\
& +\sum_{i j}\left(h_{i}^{\prime} l_{j}^{\prime} \cdot a\right) \sigma\left(h_{i}^{\prime \prime}, l_{j}^{\prime \prime}\right) \\
= & \sigma(h, l)(x y \cdot a)+\sum_{j} \sigma\left(h, l_{j}^{\prime \prime}\right)\left(x l_{j}^{\prime} \cdot a\right)+\sum_{i} \sigma\left(h_{i}^{\prime \prime}, l\right)\left(h_{i}^{\prime} y \cdot a\right) \\
& +\sum_{i j} \sigma\left(h_{i}^{\prime \prime}, l_{j}^{\prime \prime}\right)\left(h_{i}^{\prime} l_{j}^{\prime} \cdot a\right)
\end{aligned}
$$


for $a \in A$, since $A$ is an $H$-module. By the induction hypothesis, the $\sigma\left(h, l_{j}^{\prime \prime}\right)$, $\sigma\left(h_{i}^{\prime \prime}, l\right)$, and $\sigma\left(h_{i}^{\prime \prime}, l_{j}^{\prime \prime}\right)$ are all central. Hence $\sigma(h, l)$ commutes with all $x y \cdot a, a \in A$. But $G$ acts as automorphisms of $A$, so $x y \cdot A=A$. Therefore $\sigma(h, l) \in Z(A)$ and our induction is complete.

We next consider several important special cases of crossed products.

EXAMPLE 4.9. Suppose that $\sigma$ is the trivial cocycle, that is, $\sigma(h, l)=\varepsilon(h) \varepsilon(l) 1$ for all $h, l \in H$. Note that 4.5(1) is automatically satisfied so that $\sigma$ is a cocycle. Condition 4.5(2) is equivalent to $A$ being an $H$-module algebra and the multiplication in $A \#_{\sigma} H$ becomes $(a \# h)(b \# l)=\sum_{(h)(l)} a\left(h_{1} \cdot b\right) \# h_{2} l$. That is, $A \#_{\sigma} H$ is just $A \# H$, the usual smash product.

EXAMPLE 4.10. Suppose that the $H$-action is trivial (Example 1.5). We then write $A_{\sigma}[H]$ instead of $A \#_{\sigma} H$. Then condition 4.5(2) holds if and only if $\sigma(H \times H) \subseteq Z(A)$. If in addition $\sigma$ is a cocycle for the trivial action, $A_{\sigma}[H]$ is a crossed product, called the twisted product of $A$ with $H$. The multiplication in $A_{\sigma}[H]$ becomes

$$
(a \otimes h)(b \otimes l)=\sum_{(h)(l)} a b \sigma\left(h_{(1)}, l_{(1)}\right) \otimes h_{(2)} l_{(2)},
$$

where we write $a \otimes h$ instead of $a \# h$ because the $H$-action is trivial. This example extends the usual notion of twisted group algebra. If also $\sigma$ is trivial, we have $A[H]=A \otimes H$, the usual tensor product of algebras.

EXAMPLE 4.11. Suppose we are given a weak inner action of $H$ on $A$ implemented by an invertible $u \in \operatorname{Hom}_{k}(H, A)$ with $u(1)=1$ (Lemma 1.13(2)). Define $\bar{t}: H \times H \rightarrow A$ by

$$
\bar{t}(h, l)=\sum_{(h)(l)} u\left(h_{(1)}\right) u\left(l_{(1)}\right) u^{-1}\left(h_{(2)} l_{(2)}\right) .
$$

Clearly $\bar{t}$ is normal and it is easy to check that it is a cocycle (both sides of 4.5(1) reduce to

$$
\left.\sum_{(h)(l)(m)} u\left(h_{(1)}\right) u\left(l_{(1)}\right) u\left(m_{(1)}\right) u^{-1}\left(h_{(2)} l_{(2)} m_{(2)}\right)\right)
$$

and that the twisted module condition is satisfied (both sides of 4.5(2) reduce to

$$
\left.\sum_{(h)(l)} u\left(h_{(1)}\right) u\left(l_{(1)}\right) a u^{-1}\left(h_{(2)} l_{(2)}\right)\right) .
$$

Thus $A \#_{\bar{t}} H$ is a crossed product. We call $\bar{t}$ the inner cocycle defined by $u$. Corollary 5.4 will show that $A \#_{\bar{t}} H$ is algebra isomorphic to $A \otimes H$.

Our next objective is to express any Hopf algebra $H$ with Hopf homomorphic image $\bar{H}$ as a crossed product $A \#_{\sigma} \bar{H}$, where $A$ is a subalgebra of $H$. As we shall see, this can be done provided $H \rightarrow \bar{H} \rightarrow 0$ is a split coalgebra sequence.

For the remainder of this section $H \stackrel{\pi}{\rightarrow} \bar{H} \rightarrow 0$ will be an exact sequence of Hopf morphisms.

DEFinition 4.12. The (left) Hopf kernel of $\pi$ is the set

$$
\text { LHKer } \pi=\{h \in H:(\text { id } \otimes \pi) \Delta h=h \otimes \overline{1}\} .
$$


LEMMA 4.13. LHKer $\pi$ is an ad $H$-stable subalgebra of $H$.

PROOF. Let $A=$ LHKer $\pi$. Since $\Delta$ and id $\otimes \pi$ are algebra morphisms, $A$ is a subalgebra of $H$. To see that $A$ is ad $H$-stable, choose $a \in A, h \in H$ and set

$$
a^{\prime}=(\operatorname{ad} h) a=\sum_{(h)} h_{(1)} a\left(S h_{(2)}\right) .
$$

Then

$$
\Delta a^{\prime}=\sum_{(h)(a)} h_{(1)} a_{(1)}\left(S h_{(4)}\right) \otimes h_{(2)} a_{(2)}\left(S h_{(3)}\right),
$$

remembering that $\Delta S l=\sum_{(l)} S l_{(2)} \otimes S l_{(1)}$, all $l \in H$, and so

$$
(\mathrm{id} \otimes \pi) \Delta a^{\prime}=\sum_{(h)(a)} h_{(1)} a_{(1)}\left(S h_{(4)}\right) \otimes \pi\left(h_{(2)}\right) \pi\left(a_{(2)}\right) \pi\left(S h_{(3)}\right) .
$$

Using $\sum_{(a)} a_{(1)} \otimes \pi\left(a_{(2)}\right)=a \otimes \overline{1}$, this becomes

$$
\begin{aligned}
\sum_{(h)} h_{(1)} a\left(S h_{(4)}\right) \otimes \pi\left(h_{(2)}\left(S h_{(3)}\right)\right) & =\sum_{(h)} h_{(1)} a\left(S h_{(3)}\right) \otimes \pi\left(\varepsilon\left(h_{(2)}\right) 1\right) \\
& =\sum_{(h)} h_{(1)} a\left(S h_{(2)}\right) \otimes \overline{1}=a^{\prime} \otimes \overline{1}
\end{aligned}
$$

Thus $a^{\prime} \in A$, proving our contention.

THEOREM 4.14. Let $H \stackrel{\pi}{\rightarrow} \bar{H} \rightarrow 0$ be an exact sequence of Hopf algebras which is split as a coalgebra sequence; that is, there exists a coalgebra map $\gamma: \bar{H} \rightarrow H$ such that $\pi \circ \gamma=\mathrm{id}$. Suppose also $\gamma(\overline{1})=1$. Then $H \simeq A \#_{\sigma} \bar{H}$ as algebras, where $A=$ LHKer $\pi$, the weak action of $\bar{H}$ on $A$ is given by

$$
\bar{h} \cdot a=(\operatorname{ad} \gamma(\bar{h})) a \quad \text { for } \bar{h} \in \bar{H}, a \in A,
$$

and where $\sigma: \bar{H} \times \bar{H} \rightarrow A$ is given by

$$
\sigma(\bar{h}, \bar{l})=\sum_{(\bar{h})(\bar{l})} \gamma\left(\bar{h}_{(1)}\right) \gamma\left(\bar{l}_{(1)}\right)\left[S \gamma\left(\bar{h}_{(2)} \bar{l}_{(2)}\right)\right] .
$$

Proof. By Lemma 4.13, $\bar{H} \cdot A \subset A$. Now

$$
\bar{h} \cdot 1=\sum_{(\gamma(\bar{h}))} \gamma(\bar{h})_{(1)}\left[S \gamma(\bar{h})_{(2)}\right]=\varepsilon(\gamma(\bar{h})) 1=\varepsilon(\bar{h}) 1
$$

since $\varepsilon \circ \gamma=\varepsilon$, and $\overline{1} \cdot a=(\operatorname{ad} 1) a=a$ since $\gamma(\overline{1})=1$, verifying condition 1.1(2),(3). Thus $(\bar{h}, a) \mapsto \bar{h} \cdot a$ will be a weak action if we verify condition 1.1(1). But this follows from the fact that the adjoint action of $H$ on $H$ satisfies 1.1(1) and the assumption that $\gamma$ preserves comultiplication:

$$
\begin{aligned}
\bar{h} \cdot a b=(\operatorname{ad} \gamma(\bar{h})) a b & =\sum_{(\gamma(\bar{h}))}\left[\left(\operatorname{ad} \gamma(\bar{h})_{(1)}\right) a\right]\left[\left(\operatorname{ad} \gamma(\bar{h})_{(2)}\right) b\right] \\
& =\sum_{(\bar{h})}\left[\left(\operatorname{ad} \gamma\left(\bar{h}_{(1)}\right)\right) a\right]\left[\left(\operatorname{ad} \gamma\left(\bar{h}_{(2)}\right)\right) b\right]=\sum_{(\bar{h})}\left(\bar{h}_{(1)} \cdot a\right)\left(\bar{h}_{(2)} \cdot b\right) .
\end{aligned}
$$

Thus (4.15) defines a weak action of $\bar{H}$ on $A$. 
We next check that $\sigma(\bar{H} \times \bar{H}) \subseteq A$ for $\sigma$ as in (4.16). Let $\bar{h}, \bar{l} \in \bar{H}$. Then

$$
\begin{aligned}
(\mathrm{id} \otimes & \pi) \Delta \sigma(\bar{h}, \bar{l})=(\mathrm{id} \otimes \pi) \sum_{(\bar{h})(\bar{l})}\left[\Delta \gamma\left(\bar{h}_{(1)}\right)\right]\left[\Delta \gamma\left(\bar{l}_{(1)}\right)\right]\left[\Delta S \gamma\left(\bar{h}_{(2)} \bar{l}_{(2)}\right)\right] \\
= & (\operatorname{id} \otimes \pi) \sum_{(\bar{h})(\bar{l})} \gamma\left(\bar{h}_{(1)}\right) \gamma\left(\bar{l}_{(1)}\right)\left[S \gamma\left(\bar{h}_{(4)} \bar{l}_{(4)}\right)\right] \otimes \gamma\left(\bar{h}_{(2)}\right) \gamma\left(\bar{l}_{(2)}\right)\left[S \gamma\left(\bar{h}_{(3)} \bar{l}_{(3)}\right)\right] \\
= & \sum_{(\bar{h})(\bar{l})} \gamma\left(\bar{h}_{(1)}\right) \gamma\left(\bar{l}_{(1)}\right)\left[S \gamma\left(\bar{h}_{(4)} \bar{l}_{(4)}\right)\right] \otimes \bar{h}_{(2)} \bar{l}_{(2)}\left[S\left(\bar{h}_{(3)} \bar{l}_{(3)}\right)\right] \\
= & \sum_{(\bar{h})(\bar{l})} \gamma\left(\bar{h}_{(1)}\right) \gamma\left(\bar{l}_{(1)}\right)\left[S \gamma\left(\bar{h}_{(2)} \bar{l}_{(2)}\right)\right] \otimes \overline{1}=\sigma(\bar{h}, \bar{l}) \otimes \overline{1},
\end{aligned}
$$

as desired.

We may now form $A \#_{\sigma} \bar{H}$. We shall show that this is a crossed product (and hence that $\sigma$ is a normal cocycle satisfying the twisted module condition) by showing that it is algebra isomorphic to $H$, certainly an associative algebra, via a map $\Phi: A \#_{\sigma} \bar{H} \rightarrow H$ such that $\Phi(1 \# \overline{1})=1$. This will prove the theorem.

Define $\Phi$ by $\Phi(a \# \bar{h})=a \gamma(\bar{h})$ for $a \in A, \bar{h} \in \bar{H}$. Clearly $\Phi(1 \# \overline{1})=1$ since $\gamma(\overline{1})=1$. Now let $a, b \in A$ and $\bar{h}, \bar{l} \in \bar{H}$. Then

$$
\begin{aligned}
& \Phi((a \# \bar{h})(b \# \bar{l}))=\Phi\left(\sum_{(\bar{h})(\bar{l})} a\left(\bar{h}_{(1)} \cdot b\right) \sigma\left(\bar{h}_{(2)}, \bar{l}_{(1)}\right) \# \bar{h}_{(3)} \bar{l}_{(2)}\right) \\
& =\sum_{(\bar{h})(\bar{l})} a \gamma\left(\bar{h}_{(1)}\right) b\left[S \gamma\left(\bar{h}_{(2)}\right)\right] \gamma\left(\bar{h}_{(3)}\right) \gamma\left(\bar{l}_{(1)}\right)\left[S \gamma\left(\bar{h}_{(4)} \bar{l}_{(2)}\right)\right] \gamma\left(\bar{h}_{(5)} \bar{l}_{(3)}\right) .
\end{aligned}
$$

Since $\gamma$ preserves comultiplication, this collapses to $a \gamma(\bar{h}) b \gamma(\bar{l})$, that is, to $\Phi(a \# \bar{h}) \Phi(b \# \bar{l})$. We have shown that $\Phi$ is an algebra morphism sending $1 \# \overline{1}$ to 1 .

To show that $\Phi$ is bijective we will construct an inverse $\Psi: H \rightarrow A \#_{\sigma} \bar{H}$. To this end, define $Q: H \rightarrow H$ by $Q(h)=\sum_{(h)} h_{(1)}\left(S \gamma \pi h_{(2)}\right)$. We first claim that $Q(H) \subseteq A$. In fact,

$$
\begin{aligned}
(\mathrm{id} \otimes \pi) \Delta Q h & =(\mathrm{id} \otimes \pi) \sum_{(h)}\left[\Delta h_{(1)}\right]\left[\Delta S \gamma \pi h_{(2)}\right] \\
& =(\mathrm{id} \otimes \pi) \sum_{(h)} h_{(1)}\left[S \gamma \pi h_{(4)}\right] \otimes h_{(2)}\left[S \gamma \pi h_{(3)}\right] \\
& =\sum_{(h)} h_{(1)}\left[S \gamma \pi h_{(4)}\right] \otimes\left(\pi h_{(2)}\right)\left(S \pi h_{(3)}\right) \\
& =\sum_{(h)} h_{(1)}\left[S \gamma \pi h_{(4)}\right] \otimes \pi\left(h_{(2)}\left(S h_{(3)}\right)\right) \\
& =\sum_{(h)} h_{(1)}\left[S \gamma \pi h_{(2)}\right] \otimes \pi 1=(Q h) \otimes \overline{1} .
\end{aligned}
$$

Now we define $\Psi$ by $\Psi h=\sum_{(h)} Q h_{(1)} \# \pi h_{(2)}$, and we have $\Psi: H \rightarrow A \#_{\sigma} \bar{H}$. We claim that $\Phi \Psi h=h$ and $\Psi \Phi(a \# \bar{h})=a \# \bar{h}$ for all $a \in A, h \in H$, and $\bar{h} \in \bar{H}$. 
In fact,

$$
\Phi \Psi h=\sum_{(h)}\left(Q h_{(1)}\right)\left(\gamma \pi h_{(2)}\right)=\sum_{(h)} h_{(1)}\left(S \gamma \pi h_{(2)}\right)\left(\gamma \pi h_{(3)}\right)=h,
$$

because $\gamma$ and $\pi$ preserve comultiplication and counit. Moreover

$$
\begin{aligned}
\Psi \Phi(a \# \bar{h}) & =\Psi(a \gamma(\bar{h}))=\sum_{(a)(\bar{h})} Q\left(a_{(1)} \gamma\left(\bar{h}_{(1)}\right)\right) \# \pi\left(a_{(2)} \gamma\left(\bar{h}_{(2)}\right)\right) \\
& =\sum_{(a)(\bar{h})} Q\left(a_{(1)} \gamma\left(\bar{h}_{(1)}\right)\right) \#\left(\pi a_{(2)}\right) \bar{h}_{(2)}=\sum_{(\bar{h})} Q\left(a \gamma\left(\bar{h}_{(1)}\right)\right) \# \bar{h}_{(2)},
\end{aligned}
$$

since $\gamma$ preserves comultiplication and $(\mathrm{id} \otimes \pi) \Delta a=a \otimes \overline{1}$. Now

$$
\begin{aligned}
Q(a \gamma(\bar{l})) & =\sum_{(a)(\bar{l})} a_{(1)} \gamma\left(\bar{l}_{(1)}\right)\left(S \gamma\left[\left(\pi a_{(2)}\right) \pi \gamma\left(\bar{l}_{(2)}\right)\right]\right) \\
& =\sum_{(\bar{l})} a \gamma\left(\bar{l}_{(1)}\right)\left(S \gamma\left(\bar{l}_{(2)}\right)\right)=a \varepsilon(\gamma(\bar{l}))=a \varepsilon(\bar{l})
\end{aligned}
$$

for $\bar{l} \in \bar{H}$, since $\gamma$ preserves $\Delta$ and (id $\otimes \pi) \Delta a=a \otimes \overline{1}$. Substituting this into the previous equation, we get

$$
\Psi \Phi(a \# \bar{h})=\sum_{(\bar{h})} a \varepsilon\left(\bar{h}_{(1)}\right) \# \bar{h}_{(2)}=a \# \bar{h}
$$

as desired.

Since $H$ is a Hopf algebra, $A \#_{\sigma} \bar{H}$ also has a Hopf algebra structure which may be formed by pulling back $\Delta_{H}, S_{H}$ via $\Phi$. The resultant formulae are repellent, so we skip them except in the following case.

COROLlary 4.17. Assume the hypotheses of (4.14) and in addition assume that $A$ is a Hopf subalgebra of $H$. Then $H \simeq A \#_{\sigma} \bar{H}$ as Hopf algebras, where the comultiplication, counit, and antipode of $A \#_{\sigma} \bar{H}$ are given by

$$
\begin{aligned}
\Delta(a \# \bar{h}) & =\sum_{(a)(h)}\left(a_{(1)} \# \bar{h}_{(1)}\right) \otimes\left(a_{(2)} \# \bar{h}_{(2)}\right), \\
\varepsilon(a \# \bar{h}) & =\varepsilon(a) \varepsilon(\bar{h}), \text { and } \\
S(a \# \bar{h}) & =\sum_{(\bar{h})}\left(\sigma^{-1}\left(S \bar{h}_{(2)}, \bar{h}_{(3)}\right) \# S \bar{h}_{(1)}\right)(S a \# \overline{1}),
\end{aligned}
$$

where $\sigma^{-1}$, the inverse of $\sigma$ in $\operatorname{Hom}_{k}(\bar{H} \otimes \bar{H}, A)$, is given by

$$
\sigma^{-1}(\bar{h}, \bar{l})=\sum_{(\bar{h})(\bar{l})} \gamma\left(\bar{h}_{(1)} \bar{l}_{(1)}\right)\left[S \gamma\left(\bar{l}_{(2)}\right)\right]\left[S \gamma\left(\bar{h}_{(2)}\right)\right] .
$$

PROOF. Left to reader.

We remark that the expression for $S_{A \#_{\sigma} \bar{H}}$ cannot be simplified, even when $H=k G$ and $\bar{H}=k[G / N]$. The difficulty is that in general one cannot find $\gamma$ so that $\gamma \circ S=S \circ \gamma$ : in the group case, $\pi^{-1}\{\bar{x}\}$ may contain no elements of order 2 when $\bar{x}$ has order 2 . 
Corollary 4.17 raises the question of determining when $A$ is a Hopf subalgebra of $H$. Sweedler proves in [25, Lemma 16.1.1] that this is always true when $H$ is cocommutative. But this condition is not necessary. For example, let $H=A \otimes \bar{H}$, where $A$ and $\bar{H}$ are arbitrary Hopf algebras, and set $\pi(a \otimes \bar{h})=\varepsilon(a) \bar{h}$. Then $\pi$ is a Hopf algebra map with coalgebra splitting $\gamma$ given by $\gamma(\bar{h})=1 \otimes \bar{h}$. Also LHKer $\pi=A \otimes \overline{1}$, a Hopf subalgebra of $H$. The key to answering our question is:

DEFINITION 4.18. The right Hopf kernel of $\pi: H \rightarrow \bar{H}$ is the set RHKer $\pi=$ $\{h \in H:(\pi \otimes \mathrm{id}) \Delta h=\overline{\mathbf{1}} \otimes h\}$.

Clearly RHKer $\pi=$ LHKer $\pi$ if $H$ is cocommutative, and the same is true in the preceeding example. And in fact we have

Proposition 4.19. Let $A=$ LHKer $\pi$ and $A^{\prime}=$ RHKer $\pi$. Let $S$ denote the antipode of $H$. Then

(1) $S A \subseteq A^{\prime}$ and $S A^{\prime} \subseteq A$, with equality holding if $S$ is bijective.

(2) If $A=A^{\prime}$, then $A$ is a Hopf subalgebra of $H$.

(3) If $A$ is a subcoalgebra of $H$, then $A \subseteq A^{\prime}$ and $A$ is a Hopf subalgebra of $H$. Moreover, $A=A^{\prime}$ if $S$ is bijective.

Proof. (1) Recall that $\Delta \circ S=T \circ(S \otimes S) \circ \Delta$, where $T$ is the twist map $T(h \otimes l)=l \otimes h$. Thus if $a \in A$,

$$
\begin{aligned}
(\pi \otimes \mathrm{id}) \Delta S a & =(\pi \otimes \mathrm{id}) T(S \otimes S) \Delta a=T(\mathrm{id} \otimes \pi)(S \otimes S) \Delta a \\
& =T(S \otimes S)(\mathrm{id} \otimes \pi) \Delta a=T(S \otimes S)(a \otimes \overline{1})=\overline{1} \otimes S a .
\end{aligned}
$$

Thus $S a \in A^{\prime}$. Similarly $S A^{\prime} \subseteq A$.

Suppose that $S$ is bijective with inverse $\bar{S}$. The foregoing proof works for $\bar{S}$ as well as $S$, and so $\bar{S} A \subseteq A^{\prime}$ and $\bar{S} A^{\prime} \subseteq A$. Then $A^{\prime}=S \bar{S} A^{\prime} \subseteq S A$ so that $S A=A^{\prime}$, and similarly $S A^{\prime}=A$.

(2) The proof of [25, Lemma 16.1.1] actually shows that $\Delta A \subseteq H \otimes A$. Similarly $\Delta A^{\prime} \subseteq A^{\prime} \otimes H$. Thus if $A=A^{\prime}$ we conclude that $\Delta A \subseteq H \otimes A \cap A \otimes H=A \otimes A$, so $A$ is a subcoalgebra of $H$. Part (1) above then shows that $S A \subseteq A$. Thus $A$ is a Hopf subalgebra of $H$.

(3) First note that for any $a \in A, \pi a=\varepsilon(a) \overline{1}$. In fact,

$$
\begin{aligned}
\varepsilon(a) 1 \otimes \overline{1} & =(\varepsilon \otimes \mathrm{id})(a \otimes \overline{1})=(\varepsilon \otimes \mathrm{id})(\mathrm{id} \otimes \pi) \Delta a \\
& =(\mathrm{id} \otimes \pi)(\varepsilon \otimes \mathrm{id}) \Delta a=(\mathrm{id} \otimes \pi)(1 \otimes a)=1 \otimes \pi a .
\end{aligned}
$$

Since $A$ is a subcoalgebra of $H, \Delta A \subseteq A \otimes A$. Hence if $a \in A$, we have ( $\pi \otimes$ id) $\Delta a=$ $(\overline{1} \varepsilon \otimes \mathrm{id}) \Delta a=\overline{1} \otimes a$ so that $a \in A^{\prime}$. Thus $A \subseteq A^{\prime}$. But from this and (1) we deduce $S A \subseteq S A^{\prime} \subseteq A$ so that $A$ is a Hopf subalgebra of $H$. Finally, if $S$ is bijective then $A^{\prime}=S A \subseteq A$ by (1) and the previous sentence. Therefore $A=A^{\prime}$.

We now turn to three examples of the situation of Theorem 4.14. The first is the group algebra example discussed in the Introduction. The $\gamma$ there is our coalgebra splitting, the weak action there of $k \bar{G}$ on $k N$ is just given by (4.15), and the $\sigma$ defined there is just given by (4.16). Moreover, it is easy to check that LHKer $\pi=k N$, a Hopf subalgebra of $k G$.

For our next example we turn to Lie algebras.

EXAMPLE 4.20. Let $L$ be a Lie algebra over $k$ and let $N$ be a Lie ideal of $L$. Set $\bar{L}=L / N$ and consider the exact sequence of Lie algebras $L \stackrel{\pi}{\rightarrow} \bar{L} \rightarrow 0$, where $\pi$ is the canonical projection. Then $U(L) \stackrel{\pi}{\rightarrow} U(\bar{L}) \rightarrow 0$ is an exact sequence of 
Hopf algebras. Let $\left\{\bar{x}_{\alpha}\right\}$ be an ordered basis of $\bar{L}$. For each $\alpha$, choose $x_{\alpha} \in L$ such that $\pi x_{\alpha}=\bar{x}_{\alpha}$. We define $\gamma \in \operatorname{Hom}_{k}(U(\bar{L}), U(L))$ by $\gamma\left(\bar{x}_{\alpha_{1}} \cdots \bar{x}_{\alpha_{n}}\right)=x_{\alpha_{1}} \cdots x_{\alpha_{n}}$ for $\alpha_{1} \leq \alpha_{2} \leq \cdots \leq \alpha_{n}, n=0,1, \ldots$. Since $\Delta$ is an algebra homomorphism and since $\varepsilon\left(x_{\alpha_{1}} \cdots x_{\alpha_{n}}\right)=\varepsilon\left(\bar{x}_{\alpha_{1}} \cdots \bar{x}_{\alpha_{n}}\right)$ for $n \geq 1$, it is immediate that $\gamma$ is a coalgebra splitting of $U(L) \stackrel{\pi}{\rightarrow} U(\bar{L}) \rightarrow 0$. We claim that LHKer $\pi=U(N)$, a Hopf subalgebra of $U(L)$. In fact, let $a \in U(N)$. Then $\pi a=\varepsilon(a) \overline{1}$. Since $\Delta U(N) \subseteq$ $U(N) \otimes U(N)$ we have (id $\otimes \pi) \Delta a=(\mathrm{id} \otimes \overline{1} \varepsilon) \Delta a=a \otimes \overline{1}$ and hence $a \in$ LHKer $\pi$. Thus $U(N) \subseteq$ LHKer $\pi=A$, say. Now the proof of Theorem 4.14 shows that the map of $A \otimes U(\bar{L}) \rightarrow U(L)$ given by $a \otimes \bar{h} \mapsto a \gamma(\bar{h})$ is a linear isomorphism. But the Poincaré-Birkhoff-Witt Theorem says that the restriction of this map to $U(N) \otimes$ $U(\bar{L})$ maps onto $U(L)$. Therefore $A=U(N)$. (The claim can also be established by direct calculation.) Thus Corollary 4.17 says that $U(L) \simeq U(N) \#_{\sigma} U(\bar{L})$ as Hopf algebras, where the weak action of $U(\bar{L})$ on $U(N)$ is given by (4.15), $\sigma$ is given by (4.16), and the Hopf algebra structure of $U(N) \#_{\sigma} U(\bar{L})$ is given by (4.17).

Our last example is interesting because LHKer $\pi$ is not a Hopf subalgebra of $H$.

EXAMPLE 4.21. Let $G$ be a finite group with nonnormal subgroup $L$ such that there exists an algebra homomorphism $\rho: k G \rightarrow k L$ satisfying $\left.\rho\right|_{k L}=$ id. Let $H=(k G)^{*}$ and $\bar{H}=(k L)^{*}$. The map $\pi: H \rightarrow \bar{H}$ is the transpose of the injection $k L \rightarrow k G$ and hence is a surjection given by restriction of $f \in H$ to $k L$. Let $\gamma=\rho^{*}: \bar{H} \rightarrow H$. Then $\gamma$, being the transpose of an algebra map, is a coalgebra map. Moreover $\left.\rho\right|_{k L}=\mathrm{id}$ gives us that $\pi \circ \gamma=\mathrm{id}$. Thus we have an exact sequence $H \stackrel{\pi}{\rightarrow} \bar{H} \rightarrow 0$ of Hopf algebra morphisms with coalgebra splitting $\gamma$.

It is easy to check that $A=\operatorname{LHKer} \pi=\left\{f \in(k G)^{*}: f\right.$ is constant on the right cosets of $L$ in $G\}$ and $A^{\prime}=$ LHKer $\pi=\left\{f \in(k G)^{*}: f\right.$ is constant on the left cosets of $L$ in $G$ \}. Clearly $S A=A^{\prime}, S A^{\prime}=A$ (here $S$ is a bijection), but $A \neq A^{\prime}$ since $L$ is not normal in $G$. Thus $A$ is not a Hopf subalgebra of $H$ by Proposition 4.19(3). However by Theorem 4.14, it is still true that $H \simeq A \#_{\sigma} \bar{H}$.

An explicit example of such a $k, G, L$, and $\rho$ is the following: Let $k$ be a finite field of characteristic not 2 or 3 containing a primitive cube root $\omega$ of 1 , let $G=\mathrm{GL}_{2}(k)$, and let $L=S_{3}$. Now $k L$ is algebra isomorphic to $k \oplus k \oplus M_{2}(k)$, this isomorphism $\phi$ carrying $L$ into the group generated by

$$
\left(1,-1,\left(\begin{array}{ll}
0 & 1 \\
1 & 0
\end{array}\right)\right) \text { and }\left(1,1,\left(\begin{array}{cc}
\omega & 0 \\
0 & \omega^{2}
\end{array}\right)\right) \text {. }
$$

Note that $G$ is embedded into $k \oplus k \oplus M_{2}(k)$ by $x \stackrel{\psi}{\mapsto}(1, \operatorname{Det} x, x)$ so that $\psi(G) \supseteq$ $\phi(L)$. Extending $\psi$ linearly we can set $\rho=\phi^{-1} \circ \psi$. Then $k, G, L$, and $\rho$ satisfy our conditions.

5. Crossed products and inner actions. We now look more closely at crossed products in the case that the weak action of $H$ on $A$ is inner.

Let $A$ be an algebra, $H$ a Hopf algebra, and $u$ a (convolution) invertible element in $\operatorname{Hom}_{k}(H, A)$ such that $u(1)=1$. Let $\psi_{u}$ denote the inner weak action of $H$ on $A$ implemented by $u$ (Lemma 1.4). Lemma 1.13(2) shows that all inner weak actions are of the form $\psi_{u}$ for some such $u$. Let $\psi_{1}$ denote the trivial action.

Let $\sigma, \tau$ be bilinear maps from $H \times H$ to $A$ such that

$$
\tau(h, l)=\sum_{(h)(l)} u^{-1}\left(l_{(1)}\right) u^{-1}\left(h_{(1)}\right) \sigma\left(h_{(2)}, l_{(2)}\right) u\left(h_{(3)} l_{(3)}\right)
$$


for $h, l \in H$. Since $u$ is invertible, this is clearly equivalent to

$$
\sigma(h, l)=\sum_{(h)(l)} u\left(h_{(1)}\right) u\left(l_{(1)}\right) \tau\left(h_{(2)}, l_{(2)}\right) u^{-1}\left(h_{(3)} l_{(3)}\right) .
$$

As in Definition 4.2 and Example 4.10, we may form $A \#_{\sigma} H$ and $A_{\tau}[H]$ using the pairs $\left(\psi_{u}, \sigma\right)$ and $\left(\psi_{1}, \tau\right)$, respectively. The main result of this section is

THEOREM 5.3. Let $A \#_{\sigma} H$ and $A_{\tau}[H]$ be as above. Then

(1) $A \#_{\sigma} H \simeq A_{\tau}[H]$ as (possibly nonassociative) algebras (possibly without unit).

(2) $\sigma$ is normal if and only if $\tau$ is normal.

(3) $\left(\psi_{u}, \sigma\right)$ satisfies the twisted module condition 4.5(2) if and only if $\left(\psi_{1}, \tau\right)$ satisfies $4.5(2)$.

(4) If $\left(\psi_{u}, \sigma\right)$ (equivalently $\left.\left(\psi_{1}, \tau\right)\right)$ satisfies $4.5(2)$, then $\left(\psi_{u}, \sigma\right)$ satisfies the cocycle condition 4.5(1) if and only if $\left(\psi_{1}, \tau\right)$ satisfies $4.5(1)$.

(5) $A \#_{\sigma} H$ is a crossed product if and only if $A_{\tau}[H]$ is a twisted product, and they are isomorphic as algebras with 1.

Proof. (1) Let $\phi: A \#_{\sigma} H \rightarrow A_{\tau}[H]$ be defined as in Proposition 1.19. The proof there shows that $\phi$ is bijective. We check that it is a homomorphism. Let $a, b \in A, h, l \in H$. Then

$$
\begin{aligned}
\phi((a \# h)(b \# l))= & \phi\left(\sum_{(h)(l)} a\left(h_{(1)} \cdot b\right) \sigma\left(h_{(2)}, l_{(1)}\right) \# h_{(3)} l_{(2)}\right) \\
& =\sum_{(h)(l)} a\left(h_{(1)} \cdot b\right) \sigma\left(h_{(2)}, l_{(1)}\right) u\left(h_{(3)} l_{(2)}\right) \otimes h_{(4)} l_{(3)} \\
& =\sum_{(h)(l)} a u\left(h_{(1)}\right) b u^{-1}\left(h_{(2)}\right) \sigma\left(h_{(3)}, l_{(1)}\right) u\left(h_{(4)} l_{(2)}\right) \otimes h_{(5)} l_{(3)} \\
& =\sum_{(h)(l)} a u\left(h_{(1)}\right) b u\left(l_{(1)}\right) \tau\left(h_{(2)}, l_{(2)}\right) \otimes h_{(3)} l_{(3)} \\
= & \left(\sum_{(h)} a u\left(h_{(1)}\right) \otimes h_{(2)}\right)\left(\sum_{(l)} b u\left(l_{(1)}\right) \otimes l_{(2)}\right) \\
= & \phi(a \# h) \phi(b \# l),
\end{aligned}
$$

where the fourth equality follows from (5.1) together with the invertibility of $u$.

(2) is a trivial consequence of $u(1)=1$.

(3) By example 4.10, $\left(\psi_{1}, \tau\right)$ satisfies 4.5(2) if and only if $\tau(H \times H) \subseteq Z(A)$. Now the left side of equation $4.5(2)$ for $\left(\psi_{u}, \sigma\right)$ is

$$
\begin{aligned}
\sum_{(h)(l)}\left(h_{(1)} \cdot\left(l_{(1)} \cdot a\right)\right) \sigma & \left(h_{(2)}, l_{(2)}\right) \\
& =\sum_{(h)(l)} u\left(h_{(1)}\right) u\left(l_{(1)}\right) a u^{-1}\left(l_{(2)}\right) u^{-1}\left(h_{(2)}\right) \sigma\left(h_{(3)}, l_{(3)}\right) \\
& =\sum_{(h)(l)} u\left(h_{(1)}\right) u\left(l_{(1)}\right) a \tau\left(h_{(2)}, l_{(2)}\right) u^{-1}\left(h_{(3)} l_{(3)}\right),
\end{aligned}
$$


while the right side is

$$
\begin{aligned}
\sum_{(h)(l)} \sigma\left(h_{(1)}, l_{(1)}\right)\left(h_{(2)} l_{(2)} \cdot a\right) & =\sum_{(h)(l)} \sigma\left(h_{(1)}, l_{(1)}\right) u\left(h_{(2)} l_{(2)}\right) a u^{-1}\left(h_{(2)} l_{(2)}\right) \\
& =\sum_{(h)(l)} u\left(h_{(1)}\right) u\left(l_{(1)}\right) \tau\left(h_{(2)}, l_{(2)}\right) a u^{-1}\left(h_{(3)} l_{(3)}\right),
\end{aligned}
$$

using (5.1) and the invertibility of $u$. Thus 4.5(2) for $\left(\psi_{1}, \tau\right)$ implies 4.5(2) for $\left(\psi_{u}, \sigma\right)$, and the converse follows using the invertibility of $u$.

(4) The left side of $4.5(1)$ for $\left(\psi_{u}, \sigma\right)$ is

$$
\begin{aligned}
& \sum_{(h)(l)(m)}\left[h_{(1)} \cdot \sigma\left(l_{(1)}, m_{(1)}\right)\right] \sigma\left(h_{(2)}, l_{(2)} m_{(2)}\right) \\
& \quad=\sum_{(h)(l)(m)} u\left(h_{(1)}\right) \sigma\left(l_{(1)}, m_{(1)}\right) u^{-1}\left(h_{(2)}\right) \sigma\left(h_{(3)}, l_{(2)} m_{(2)}\right) \\
& \quad=\sum_{(h)(l)(m)} u\left(h_{(1)}\right) u\left(l_{(1)}\right) u\left(m_{(1)}\right) \tau\left(l_{(2)}, m_{(2)}\right) \tau\left(h_{(2)}, l_{(3)} m_{(3)}\right) u^{-1}\left(h_{(3)} l_{(4)} m_{(4)}\right)
\end{aligned}
$$

while the right side is

$$
\begin{aligned}
& \sum_{(h)(l)(m)} \sigma\left(h_{(1)}, l_{(1)}\right) \sigma\left(h_{(2)} l_{(2)}, m\right) \\
& \quad=\sum_{(h)(l)(m)} u\left(h_{(1)}\right) u\left(l_{(1)}\right) \tau\left(h_{(2)}, l_{(2)}\right) u\left(m_{(1)}\right) \tau\left(h_{(3)} l_{(3)}, m_{(2)}\right) u^{-1}\left(h_{(4)} l_{(4)} m_{(3)}\right)
\end{aligned}
$$

using (5.2) and the invertibility of $u$. Since 4.5(2) is true for $\left(\psi_{1}, \tau\right), \tau(H \times H) \subseteq$ $Z(A)$, and the right side of $4.5(1)$ for $\left(\psi_{u}, \sigma\right)$ becomes

$$
\sum_{(h)(l)(m)} u\left(h_{(1)}\right) u\left(l_{(1)}\right) u\left(m_{(1)}\right) \tau\left(h_{(2)}, l_{(2)}\right) \tau\left(h_{(3)} l_{(3)}, m_{(2)}\right) u^{-1}\left(h_{(4)} l_{(4)} m_{(3)}\right) .
$$

Now $4.5(1)$ for $\left(\psi_{1}, \tau\right)$ is just

$$
\sum_{(l)(m)} \tau\left(l_{(1)}, m_{(1)}\right) \tau\left(h, l_{(2)} m_{(2)}\right)=\sum_{(h)(l)} \tau\left(h_{(1)}, l_{(1)}\right) \tau\left(h_{(2)} l_{(2)}, m\right)
$$

so that this yields 4.5(1) for $\left(\psi_{u}, \sigma\right)$. Conversely, 4.5(1) for $\left(\psi_{u}, \sigma\right)$ yields $4.5(1)$ for $\left(\psi_{1}, \tau\right)$, using the invertibility of $u$.

(5) follows from (1)-(4) and Corollary 4.6.

Here are two special cases to which Theorem 5.3 applies.

COROLLARY 5.4. Let $\bar{t}$ be the inner cocycle attached to the weak inner action $\psi_{u}$ as in Example 4.11. Then $A \#_{\bar{t}} H \simeq A \otimes H$.

Proof. Let $\tau$ be trivial in Theorem 5.3 and note that then $\sigma=\bar{t}$ and $A_{\tau}[H]=$ $A \otimes H$.

COROLlaRY 5.5. Let $u$ implement an inner action on $A$ and define $t$ as in Definition 1.3. Then $A \# H \simeq A_{t}[H]$. Moreover, $A_{t}[H]=A \otimes H$ if and only if $u \in \operatorname{Alg}(H, A)$.

ProOF. Applying Theorem 5.3 to the case where $\sigma$ is trivial, and noting that $\tau=t$, gives our first assertion. Since $u$ is invertible, $t$ is trivial if and only if $u \in$ $\operatorname{Alg}(H, A)$. If $t$ is trivial, then plainly $A_{t}[H]=A \otimes H$. Conversely, if $A_{t}[H]=A \otimes H$, 
then

$$
\sum_{(h)(l)} t\left(h_{(1)}, l_{(1)}\right) \otimes h_{(2)} l_{(2)}=(1 \otimes h)(1 \otimes l)=1 \otimes h l
$$

for all $h, l \in H$. Applying id $\otimes \varepsilon$ to both sides, we obtain $t(h, l)=\varepsilon(h l) 1$, so that $t$ is trivial. This yields the second assertion.

Thus once again we obtain Proposition 1.19. The first assertion of Corollary 5.5 was already known for $H=k G$ (see [16, p. 107]). The correct setting for the sort of things considered in Theorem 5.3 and its corollaries is the nonabelian cohomology theory of Hopf algebras. This will be taken up in a later paper.

Several times in what follows we will make use of the fact that for any weak action of $H$ on $A$ and any bilinear map $\sigma: H \times H \rightarrow A$, the space $A \#_{\sigma} H$ is a right $H$-comodule algebra via the map

$$
\rho=\mathrm{id} \otimes \Delta: A \#_{\sigma} H \rightarrow\left(A \#_{\sigma} H\right) \otimes H
$$

that is, $\rho$ satisfies (3) and (4) of Definition 2.1. (Indeed, the weak action and bilinear map play no part in this fact.)

Let $A$ be a strongly inner $G$-graded algebra. Then Proposition 1.19 says that $A \#(k G)^{0} \simeq A \otimes(k G)^{0}$. Our next example shows that we cannot weaken the hypothesis to only requiring $A$ to be inner graded. First we need a slight strengthening of [4, Theorem 3.2].

PROPOSITION 5.6 (DUALITY FOR TWISTED GROUP ACTIONS). Let $B \#_{\sigma}(k G)$ be a crossed product with $|G|=n<\infty$. Then $B \#_{\sigma}(k G)$ is graded by $G$ in the usual way and

$$
\left(B \#_{\sigma}(k G)\right) \#(k G)^{*} \simeq B \otimes M_{n}(k) .
$$

PROOF. Exactly the same proof given in [4] works here.

Note that the $G$-grading of $B \#_{\sigma}(k G)$ is exactly that arising from the right $k G$-comodule structure on $B \#_{\sigma}(k G)$ discussed above. Also note that the proof of duality for coactions of finite groups in [4] does not carry over to the twisted case.

EXAMPLE 5.7. Let $A=C_{2}(k)$ as in Example 3.6. In the notation of that example, $A=k_{\tau}[G]$, that is, $A=k \#_{\tau}(k G)$ with trivial $G$-action on $k$. The $G$ grading of $A$ was shown to be inner but not strongly inner. Now $(k G)^{*}$ acts on $A$, and we have $A \#(k G)^{*} \simeq k \otimes M_{4}(k)=M_{4}(k)$ by Proposition 5.6. On the other hand, $A \otimes(k G)^{*} \simeq A^{4}=C_{2}(k)^{4}$, since $(k G)^{*}$ is a direct sum of four copies of $k$. Thus $A \#(k G)^{*} \not A \otimes(k G)^{*}$ even though the $G$-grading is inner.

We now turn to possible twisted analogues of Theorem 1.22. First note that since $A \#_{\sigma} H$ is a right $H$-comodule, it is a left $H^{0}$-module, and one can check that if $A \#_{\sigma} H$ is a crossed product, it is an $H^{0}$-module algebra. Now the direct analogue of Theorem 1.22 is false. For, consider Example 3.6 again. Then $k_{\tau}[G]=k \#_{\tau}(k G)$, where the action of $k G$ on $k$ is trivial and so is certainly strongly inner. However, the action of $(k G)^{*}$ on $k \#_{\tau}(k G)$ is inner and not outer.

It $i s$ true in Example 3.6 that the action of $(k G)^{*}$ on $k \#_{\tau}(k G)$ is not strongly inner. This fact can be generalized to general crossed products as follows.

THEOREM 5.8. Let $H$ be a nontrivial residually finite dimensional cocommutative Hopf algebra and let $A \#_{\sigma} H$ be a crossed product. If $H^{0}$ is strongly inner on $A \#_{\sigma} H$, then $H$ is outer on $A$. 
To prove Theorem 5.8, we require three lemmas, the first of which depends on Theorem 5.3.

LEMmA 5.9. If $\operatorname{Cent}_{A \#_{\sigma} H}\left(A \#_{\sigma} 1\right) \subseteq A \#_{\sigma} 1$, and $H$ is not trivial, then $H$ is outer on $A$.

ProOF. Let $L$ be a Hopf subalgebra of $H$ which is inner on $A$. Then Theorem 5.3 implies that $A \#_{\sigma} L \simeq A_{\tau}[L]$ via a map $\phi$ such that $\phi(a \# 1)=a \otimes 1$. So applying $\phi$ to our hypothesis that $\operatorname{Cent}_{A \#_{\sigma} H}(A \# 1) \subseteq A \# 1$, we obtain Cent $_{A_{\tau}[H]}(A \otimes 1) \subseteq A \otimes 1$. But in $A_{\tau}[L], 1 \otimes L$ centralizes $A \otimes 1$ since $\tau$ is normal and the action of $L$ on $A$ is trivial. Thus $1 \otimes L \subseteq A \otimes 1$, so that $L$ must be trivial.

Recall that the set of invariants of any Hopf algebra $K$ acting on an algebra $B$ is the set $B^{K}=\{b \in B: h \cdot b=\varepsilon(h) b$, all $h \in K\}$.

LEMMA 5.10. If $H$ is residually finite dimensional, then

$$
\left(A \#_{\sigma} H\right)^{H^{0}}=A \#_{\sigma} 1 .
$$

ProOF. By hypothesis, $H^{0}$ is dense in $H^{*}$. Using (2.5), it follows that

$$
\left(A \#_{\sigma} H\right)^{H^{0}}=\left\{b \in A \#_{\sigma} H: \rho b=b \otimes 1\right\},
$$

where

$$
\rho=\mathrm{id} \otimes \Delta: A \#_{\sigma} H \rightarrow\left(A \#_{\sigma} H\right) \otimes H
$$

is the right $H$-comodule structure on $A \#_{\sigma} H$. Clearly $A \#_{\sigma} 1 \subseteq\left(A \#_{\sigma} H\right)^{H^{0}}$. Conversely, suppose $\rho b=b \otimes 1$. Then $b=(\mathrm{id} \otimes \varepsilon \otimes \mathrm{id}) \rho b=(\mathrm{id} \otimes \varepsilon \otimes \mathrm{id})(b \otimes 1) \epsilon$ $A \#_{\sigma} 1$, as desired.

LEMMA 5.11. Suppose that the action of $K$ on $B$ is inner, implemented by $u \in$ $\operatorname{Hom}(K, B)$. Then $u(K)$ is a commutative subset of $B$ if and only if $\operatorname{Cent}_{B}\left(B^{K}\right) \subseteq$ $B^{K}$.

ProOF. Suppose that $b \in \operatorname{Cent}_{B}(u(K))$. Then

$$
h \cdot b=\sum_{(h)} u\left(h_{(1)}\right) b u^{-1}\left(h_{(2)}\right)=\sum_{(h)} b u\left(h_{(1)}\right) u^{-1}\left(h_{(2)}\right)=\varepsilon(h) b
$$

for all $h \in K$, so that $b \in B^{K}$. Suppose, conversely, that $b \in B^{K}$. Then by Lemma 1.14

$$
u(h) b=\sum_{(h)}\left(h_{(1)} \cdot b\right) u\left(h_{(2)}\right)=\sum_{(h)} \varepsilon\left(h_{(1)}\right) b u\left(h_{(2)}\right)=b u(h)
$$

for all $h \in K$, that is, $b \in \operatorname{Cent}_{B}(u(K))$. So we have shown that $\operatorname{Cent}_{B}(u(K))=$ $B^{K}$. Suppose that $u(K)$ is commutative. Then $u(K) \subseteq \operatorname{Cent}_{B}(u(K))=B^{K}$, which implies that $\operatorname{Cent}_{B}\left(B^{K}\right) \subseteq \operatorname{Cent}_{B}(u(K))=B^{K}$. Conversely, if $\operatorname{Cent}_{B}\left(B^{K}\right) \subseteq B^{K}$, then $u(K) \subseteq \operatorname{Cent}_{B}\left(\operatorname{Cent}_{B}(u(K))\right)=\operatorname{Cent}_{B}\left(B^{K}\right) \subseteq B^{K}=\operatorname{Cent}_{B}(u(K))$ and so $u(K)$ is commutative.

Proof of ThEOREM 5.8. Let $K=H^{0}$ be strongly inner on $B=A \#_{\sigma} H$, implemented by $u \in \operatorname{Alg}(K, B)$. Since $H$ is cocommutative, $K$ is commutative and hence so is $u(K)$. By Lemma 5.11, Cent $_{B}\left(B^{K}\right) \subseteq B^{K}$. But by Lemma 5.10, $B^{K}=A \#_{\sigma} 1$ and so we have $\operatorname{Cent}_{A \#_{\sigma} H}\left(A \#_{\sigma} 1\right) \subseteq A \#_{\sigma} 1$. Then Lemma 5.9 says that $H$ is outer on $A$.

There is one simple situation in which the direct analogue of Theorem 1.22 does hold for crossed products: when $\operatorname{dim} H=2$. 
CoROllaRY 5.12. If $\operatorname{dim} H=2$ and $H$ is inner on $A$, then $H^{*}$ is outer on $A \#_{\sigma} H$.

Proof. If $H^{*}$ is not outer on $A \#_{\sigma} H$, then it is inner, since $H^{*}$ has no proper Hopf subalgebras. So let the inner action of $H^{*}$ on $A \#_{\sigma} H$ be implemented by $u \in \operatorname{Hom}\left(H^{*}, A \#_{\sigma} H\right)$. But then $u\left(H^{*}\right)$ is commutative since it is spanned by $1=u(1)$ and one other element of $A \#_{\sigma} H$. As in the proof of Theorem 5.8, Lemmas 5.9-5.11 imply that the weak action of $H$ on $A$ is outer, a contradiction.

We conclude this section with two results which relate inner actions to right-left symmetry conditions of the sort considered in [2]. The set-up is as follows: Let $H$ be a Hopf algebra with bijective antipode, and let $U$ be a Hopf subalgebra of $H^{0}$ with bijective antipode. Let $\bar{S}$ denote the (ordinary!) inverse of the antipode of $U$. The homomorphism $\lambda: H \# U \rightarrow \operatorname{End}_{k} H$ defined by $\lambda(h \# f) l=h(f-l)$ for $h, l \in$ $H, f \in U$, is injective [2, Corollary 2.3(i)]. There is also an antihomomorphism $\rho: U \# H \rightarrow \operatorname{End}_{k} H$ defined by $\rho(f \# h) l=(l \leftarrow f) h=\sum_{(l)}\left\langle f, l_{(1)}\right\rangle l_{(2)} h$. The RL-condition on $H$ and $U$ [2, Definition 1.3] is $\rho(U \# 1) \subseteq \lambda(H \# U)$. We first show that the RL-condition is closely related to the innerness of the weak action $\psi$ of $U$ on $H \# U$ given by

$$
g \cdot(h \# f)=(h-g) \# f=(\rho(g \# 1) h) \# f .
$$

Proposition 5.13. The RL-condition implies that $\psi$ is inner. The converse holds provided $\operatorname{Cent}_{\operatorname{End}_{k} H} \lambda(H \# U)$ is scalar (e.g. if $U$ is dense in $H^{*}$ ).

PROOF. We first show that our weak action is always inner in $\operatorname{End}_{k} H$, in fact that $\lambda(g \cdot b)=(\operatorname{ad} \rho(g \# 1)) \lambda(b)$ for all $g \in U, b \in H \# U$. Let $h, l \in H, f, g \in U$. Then

$$
\begin{aligned}
\sum_{(g)} \rho & \left(g_{(1)} \# 1\right) \lambda(h \# f) \rho\left(\bar{S} g_{(2)} \# 1\right) l \\
= & \sum_{(g)(l)}\left\langle\bar{S} g_{(2)}, l_{(1)}\right\rangle \rho\left(g_{(1)} \# 1\right) \lambda(h \# f) l_{(2)} \\
= & \sum_{(g)(l)}\left\langle\bar{S} g_{(2)}, l_{(1)}\right\rangle\left\langle f, l_{(3)}\right\rangle \rho\left(g_{(1)} \# 1\right) h l_{(2)} \\
= & \sum_{(g)(h)(l)}\left\langle\bar{S} g_{(2)}, l_{(1)}\right\rangle\left\langle f, l_{(4)}\right\rangle\left\langle g_{(1)}, h_{(1)} l_{(2)}\right\rangle h_{(2)} l_{(3)} \\
= & \sum_{(g)(h)(l)}\left\langle\bar{S} g_{(3)}, l_{(1)}\right\rangle\left\langle f, l_{(4)}\right\rangle\left\langle g_{(1)}, h_{(1)}\right\rangle\left\langle g_{(2)}, l_{(2)}\right\rangle h_{(2)} l_{(3)} \\
= & \sum_{(g)(h)(l)}\left\langle\left(\bar{S} g_{(3)}\right) g_{(2)}, l_{(1)}\right\rangle\left\langle f, l_{(3)}\right\rangle\left\langle g_{(1)}, h_{(1)}\right\rangle h_{(2)} l_{(2)} \\
= & \sum_{(g)(h)(l)}\left\langle\varepsilon\left(g_{(2)}\right) 1, l_{(1)}\right\rangle\left\langle f, l_{(3)}\right\rangle\left\langle g_{(1)}, h_{(1)}\right\rangle h_{(2)} l_{(2)} \\
= & \sum_{(h)(l)}\left\langle f, l_{(2)}\right\rangle\left\langle g, h_{(1)}\right\rangle h_{(2)} l_{(1)}=(h-g)(f-l)=\lambda(g \cdot(h \# f)) l
\end{aligned}
$$

as desired. (Note that setting $h \# f=1 \# 1$ shows that $g \mapsto \rho(\bar{S} g \# 1)$ is the convolution inverse of $g \mapsto \rho(g \# 1)$ in $\operatorname{Hom}_{k}\left(U, \operatorname{End}_{k} H\right)$.) 
Suppose that the RL-condition holds. Then $\rho(g \# 1) \subseteq \lambda(H \# U)$ so that there exists a unique $u(g) \in H \# U$ such that $\rho(g \# 1)=\lambda(u(g))$. Then the foregoing shows that $\sum_{(g)} u\left(g_{(1)}\right)(h \# f) u\left(\bar{S} g_{(2)}\right)=g \cdot(h \# f)$ so that the weak $U$-action is inner.

Conversely, suppose that the weak action is inner, implemented by

$$
u \in \operatorname{Hom}(U, H \# U) \text {. }
$$

Applying $\lambda$, we see that $(\operatorname{ad} \rho(g \# 1)) \lambda(b)=(\operatorname{ad} \lambda(u(g))) \lambda(b)$ for all $g \in U, b \in$ $H \# U$. Set $\bar{\rho}(g)=\rho(g \# 1)$ and $c=\left(\lambda \circ u^{-1}\right) * \bar{\rho}$ in $\operatorname{Hom}\left(U, \operatorname{End}_{k} H\right)$. Then $c(g)$ centralizes $\lambda(H \# U)$ for all $g \in H^{0}$ and so is a scalar by our centralizer hypothesis. Thus $\bar{\rho}=(\lambda \circ u) * c$ so that $\bar{\rho}(U) \subseteq \lambda(H \# U)$, which is the RL-condition.

We remark that if $H$ is cocommutative, the RL-condition is always satisfied and we are back to Example 2.12. Our second result uses a different right-left symmetry condition and relates it to the standard action of $H$ on $H$ \#, viz.

$$
l \cdot(h \# f)=h \#(l \rightarrow f)
$$

for $h, l \in H, f \in U$.

PROPOSITION 5.14. If $\rho(1 \# H) \subseteq \lambda(H \# U)$, then the standard action of $H$ on $H \# U$ is inner. If $\operatorname{Cent}_{\operatorname{End}_{k} H} \lambda(H \# U)$ is scalar, then the converse holds. Moreover, if the standard action is inner, it is strongly inner.

ProOF. One shows, as in the proof of Proposition 5.13, that

$$
\lambda(l \cdot b)=(\operatorname{ad} \rho(1 \# \bar{S} l)) \lambda(b)
$$

for $l \in H, b \in H \# U$ and then proceeds as before. The last assertion follows from the fact that $l \mapsto \rho(1 \# \bar{S} l)$ belongs to $\operatorname{Alg}\left(H, \operatorname{End}_{k} H\right)$.

COROLlary 5.15. If $\operatorname{dim} H<\infty$, then the weak action of $H^{*}$ on $H \# H^{*}$ is inner and the standard action of $H$ on $H \# H^{*}$ is strongly inner.

6. Maschke-type theorems for crossed products. The classical version of Maschke's theorem states that for a finite group $G$, the group algebra $k G$ is semisimple if and only if $|G|^{-1} \in k$. This has been generalized to crossed products of groups over rings in several ways. Consider $A \#_{\sigma}(k G)=A * G$, where $|G|^{-1} \in k$. Standard arguments show that if $V$ is a (left) $A * G$ module which is completely reducible as an $A$-module, then it is completely reducible as an $A * G$-module (see for example [13]). As a consequence of this, if $A$ is semisimple, then $A * G$ is semisimple, since if $W$ is a faithful completely reducible $A$-module, then $\bar{W}=(A * G) \otimes_{A} W=$ $\sum_{g \in G} g \otimes W$ is a faithful completely reducible $A * G$-module. A more general such result is a theorem of Fisher and Montgomery [16, p. 109]: if $A$ is semiprime, then $A * G$ is semiprime.

We are concerned in this section with to what extent analogs of these results can be proved for $A \#_{\sigma} H$, where $H$ is a finite dimensional semisimple Hopf algebra. Such results are known when $\sigma$ is trivial and $H=(k G)^{*}$ or $u(L)$, the (restricted) enveloping algebra of a finite dimensional restricted Lie algebra; more generally, 
when $H$ is commutative $[\mathbf{4}, \mathbf{1}]$. For arbitrary semisimple $H$, Cohen and Fishman have proved the first of the above results when $\sigma$ is trivial [3], based on an argument of Larson and Sweedler [11]:

Proposition 6.1. Let $H$ be finite dimensional semisimple and $A$ an $H$-module algebra. If $V$ is a (left) $A$ \# H-module which is completely reducible as an $A$-module, then it is completely reducible as an $A \# H$-module.

An immediate consequence of Proposition 6.1 is that if $A$ is semisimple Artinian, then so is $A \# H[3]$. However, the corresponding question for semiprimeness remains open.

In this section, we first show that Proposition 6.1 extends to crossed products, under the additional hypotheses that $H$ is cocommutative and $\sigma$ is invertible in $\operatorname{Hom}_{k}(H \otimes H, A)$. We then consider the semiprimeness question and show that it holds in the following two cases.

(1) Any $A \# H$, provided the action of $H$ is inner.

(2) Any $A_{\sigma}[H]$, provided $H$ is cocommutative and $\sigma$ is invertible.

To begin, consider any crossed product $A \#_{\sigma} H$ such that $\sigma$ is invertible. Define $\gamma \in \operatorname{Hom}\left(H, A \#_{\sigma} H\right)$ by $\gamma(h)=1 \# h$, and define $\mu \in \operatorname{Hom}\left(H, A \#_{\sigma} H\right)$ by

$$
\mu(h)=\sum_{(h)} \sigma^{-1}\left(S h_{(2)}, h_{(3)}\right) \# S h_{(1)} .
$$

LEMMA 6.2. (1) $\mu$ is a left convolution inverse for $\gamma$.

(2) If $H$ is cocommutative, then $\mu$ is also a right convolution inverse for $\gamma$. Moreover, $A \#_{\sigma} H=\left(A \#_{\sigma} 1\right) \mu(H)$.

Proof. Let $h \in H$. Then

$$
\begin{aligned}
(\mu * \gamma)(h) & =\sum_{(h)}\left(\sigma^{-1}\left(S h_{(2)}, h_{(3)}\right) \# S h_{(1)}\right)\left(1 \# h_{(4)}\right) \\
& =\sum_{(h)} \sigma^{-1}\left(S h_{(3)}, h_{(4)}\right) \sigma\left(S h_{(2)}, h_{(5)}\right) \#\left(S h_{(1)}\right) h_{(6)} \\
& =\sum_{(h)} \varepsilon\left(S h_{(2)}\right) \varepsilon\left(h_{(3)}\right) 1 \#\left(S h_{(1)}\right) h_{(4)} \\
& =\sum_{(h)} 1 \#\left(S h_{(1)}\right) h_{(2)}=\varepsilon(h) 1 \# 1 .
\end{aligned}
$$

Thus $\mu$ is a left inverse for $\gamma$.

(2) Let $h \in H$. Then

$$
\begin{aligned}
(\gamma * \mu)(h) & =\sum_{(h)}\left(1 \# h_{(1)}\right)\left(\sigma^{-1}\left(S h_{(3)}, h_{(4)}\right) \# S h_{(2)}\right) \\
& =\sum_{(h)}\left[h_{(1)} \cdot \sigma^{-1}\left(S h_{(6)}, h_{(7)}\right)\right] \sigma\left(h_{(2)}, S h_{(5)}\right) \# h_{(3)}\left(S h_{(4)}\right) \\
& =\sum_{(h)}\left[h_{(1)} \cdot \sigma^{-1}\left(S h_{(4)}, h_{(5)}\right)\right] \sigma\left(h_{(2)}, S h_{(3)}\right) \# 1 .
\end{aligned}
$$


Thus we wish to show that

$$
\sum_{(h)}\left[h_{(1)} \cdot \sigma^{-1}\left(S h_{(4)}, h_{(5)}\right)\right] \sigma\left(h_{(2)}, S h_{(3)}\right)=\varepsilon(h) 1 .
$$

Now for any Hopf algebra $H$, if $\sigma$ is a normal cocycle, we have

$$
\begin{gathered}
\sum_{(h)}\left[h_{(1)} \cdot \sigma\left(S h_{(4)}, h_{(5)}\right)\right] \sigma\left(h_{(2)},\left(S h_{(3)}\right) h_{(6)}\right)=\sum_{(h)} \sigma\left(h_{(1)}, S h_{(4)}\right) \sigma\left(h_{(2)}\left(S h_{(3)}\right), h_{(5)}\right) \\
=\sum_{(h)} \sigma\left(h_{(1)}, S h_{(3)}\right) \varepsilon\left(h_{(2)}\right) \varepsilon\left(h_{(4)}\right)=\sum_{(h)} \sigma\left(h_{(1)}, S h_{(2)}\right) .
\end{gathered}
$$

When $H$ is cocommutative, the left side of this equation becomes

$$
\begin{aligned}
& \sum_{(h)}\left[h_{(1)} \cdot \sigma\left(S h_{(2)}, h_{(3)}\right)\right] \sigma\left(h_{(4)},\left(S h_{(5)}\right) h_{(6)}\right) \\
& \quad=\sum_{(h)}\left[h_{(1)} \cdot \sigma\left(S h_{(2)}, h_{(3)}\right)\right] \varepsilon\left(h_{(4)}\right) \varepsilon\left(h_{(5)}\right)=\sum_{(h)} h_{(1)} \cdot \sigma\left(S h_{(2)}, h_{(3)}\right) .
\end{aligned}
$$

Substituting this, with shift of subscript, for the $\sigma\left(h_{(2)}, S h_{(3)}\right)$ factor in the left side of $(*)$, we get

$$
\begin{gathered}
\sum_{(h)}\left[h_{(1)} \cdot \sigma^{-1}\left(S h_{(5)}, h_{(6)}\right)\right]\left[h_{(2)} \cdot \sigma\left(S h_{(3)}, h_{(4)}\right)\right] \\
=\sum_{(h)} h_{(1)} \cdot\left[\sigma^{-1}\left(S h_{(4)}, h_{(5)}\right) \sigma\left(S h_{(2)}, h_{(3)}\right)\right] \\
=\sum_{(h)} h_{(1)} \cdot\left[\varepsilon\left(S h_{(2)}\right) \varepsilon\left(h_{(3)}\right) 1\right]=\varepsilon(h) 1,
\end{gathered}
$$

as desired. (Cocommutativity was used again for the second to last equality.) Thus $\mu$ is also a right inverse for $\gamma$, and so $\mu=\gamma^{-1}$.

Since $H$ is cocommutative, $S H=H$, and thus to show that $A \#_{\sigma} H=$ $\left(A \#_{\sigma} 1\right) \mu(H)$ it will suffice to check that $1 \# S h \in\left(A \#_{\sigma} 1\right) \mu(H)$ for any $h \in H$. Now

$$
\begin{aligned}
1 \# S h & =\sum_{(h)} \varepsilon\left(S h_{(1)}\right) \varepsilon\left(h_{(2)}\right) \# S h_{(3)} \\
& =\sum_{(h)} \sigma\left(S h_{(2)}, h_{(3)}\right) \sigma^{-1}\left(S h_{(1)}, h_{(4)}\right) \# S h_{(5)} \\
& =\sum_{(h)} \sigma\left(S h_{(1)}, h_{(2)}\right) \sigma^{-1}\left(S h_{(4)}, h_{(5)}\right) \# S h_{(3)} \\
& =\sum_{(h)}\left(\sigma\left(S h_{(1)}, h_{(2)}\right) \# 1\right) \mu\left(h_{(3)}\right),
\end{aligned}
$$

where cocommutativity was used again for the second to last equality.

LEMMA 6.3. Let $H$ be a finite dimensional Hopf algebra, and let $x$ be a left integral in $H$ (i.e. $x h=\varepsilon(h) x$, all $h \in H$ ). Let $A \#_{\sigma} H$ be a crossed product 
with $\sigma$ invertible, $\gamma$ invertible, and $A \#_{\sigma} H=\left(A \#_{\sigma} 1\right) \gamma^{-1}(H)$. Let $V, W$ be left $A \#_{\sigma} H$-modules and let $\lambda \in \operatorname{Hom}_{A \#_{\sigma} 1}(V, W)$. Define $\tilde{\lambda}: V \rightarrow W$ by

$$
\tilde{\lambda}(v)=\sum_{(x)} \gamma^{-1}\left(x_{(1)}\right) \cdot \lambda\left(\gamma\left(x_{(2)}\right) v\right),
$$

for all $v \in V$. Then $\tilde{\lambda} \in \operatorname{Hom}_{A \#_{o} H}(V, W)$.

Proof. For ease of notation, we will regard $A$ as embedded in $A \#_{\sigma} H$ and write $a$ in place of $a \# 1, a \in A$.

Since $A \#_{\sigma} H=(A \# 1) \gamma^{-1}(H)$, it suffices to show that $\tilde{\lambda}$ is an $A$-module map and that

$$
\tilde{\lambda}\left(\gamma^{-1}(h) v\right)=\gamma^{-1}(h) \tilde{\lambda}(v),
$$

all $h \in H, v \in V$.

We first check that $\tilde{\lambda}$ is an $A$-module map. Since $\gamma(h) a=\sum_{(h)}\left(h_{(1)} \cdot a\right) \gamma\left(h_{(2)}\right)$, any $h \in H$, and since $\gamma$ is invertible, it follows that

$$
h \cdot a=\sum_{(h)} \gamma\left(h_{(1)}\right) a \gamma^{-1}\left(h_{(2)}\right) .
$$

Thus for any $a \in A$,

$$
\begin{aligned}
\tilde{\lambda}(a v) & =\sum_{(x)} \gamma^{-1}\left(x_{(1)}\right) \lambda\left(\gamma\left(x_{(2)}\right) a v\right) \\
& =\sum_{(x)} \gamma^{-1}\left(x_{(1)}\right) \lambda\left(\left(x_{(2)} \cdot a\right) \gamma\left(x_{(3)}\right) v\right) \\
& =\sum_{(x)} \gamma^{-1}\left(x_{(1)}\right)\left(x_{(2)} \cdot a\right) \lambda\left(\gamma\left(x_{(3)}\right) v\right) \\
& =\sum_{(x)} \gamma^{-1}\left(x_{(1)}\right) \gamma\left(x_{(2)}\right) a \gamma^{-1}\left(x_{(3)}\right) \lambda\left(\gamma\left(x_{(4)}\right) v\right) \\
& =\sum_{(x)} \varepsilon\left(x_{(1)}\right) a \gamma^{-1}\left(x_{(2)}\right) \lambda\left(\gamma\left(x_{(3)}\right) v\right) \\
& =a \sum_{(x)} \gamma^{-1}\left(x_{(1)}\right) \lambda\left(\gamma\left(x_{(2)}\right) v\right)=a \tilde{\lambda}(v) .
\end{aligned}
$$

To check that $\tilde{\lambda}\left(\gamma^{-1}(h) v\right)=\gamma^{-1}(h) \tilde{\lambda}(v)$, we need the fact that $x$ is a left integral: that is, $x h=\varepsilon(h) x$, for all $h \in H$. It follows that

$$
\begin{aligned}
(\Delta x) \otimes h & =\sum_{(h)} \Delta\left(\varepsilon\left(h_{(1)}\right) x\right) \otimes h_{(2)} \\
& =\sum_{(h)} \Delta\left(x h_{(1)}\right) \otimes h_{(2)} \\
& =\sum_{(h)} x_{(1)} h_{(1)} \otimes x_{(2)} h_{(2)} \otimes h_{(3)} .
\end{aligned}
$$


Then

$$
\begin{aligned}
\tilde{\lambda}\left(\gamma^{-1}(h) v\right) & =\sum_{(x)} \gamma^{-1}\left(x_{(1)}\right) \lambda\left(\gamma\left(x_{(2)}\right) \gamma^{-1}(h) v\right) \\
& =\sum_{(x)(h)} \gamma^{-1}\left(x_{(1)} h_{(1)}\right) \lambda\left(\gamma\left(x_{(2)} h_{(2)}\right) \gamma^{-1}\left(h_{(3)}\right) v\right) \\
& =\sum_{(x)(h)} \gamma^{-1}\left(x_{(1)} h_{(1)}\right) \lambda\left[\sigma^{-1}\left(x_{(2)}, h_{(2)}\right) \gamma\left(x_{(3)}\right) \gamma\left(h_{(3)}\right) \cdot \gamma^{-1}\left(h_{(4)}\right) v\right] \\
& =\sum_{(x)(h)} \gamma^{-1}\left(x_{(1)} h_{(1)}\right) \lambda\left[\sigma^{-1}\left(x_{(2)}, h_{(2)}\right) \gamma\left(x_{(3)}\right) \varepsilon\left(h_{(3)}\right) v\right] \\
& =\sum_{(x)(h)} \gamma^{-1}\left(x_{(1)} h_{(1)}\right) \sigma^{-1}\left(x_{(2)}, h_{(2)}\right) \lambda\left(\gamma\left(x_{(3)}\right) v\right) \\
& =\sum_{(x)(h)}\left[\gamma^{-1}\left(h_{(1)}\right) \gamma^{-1}\left(x_{(1)}\right) \sigma\left(x_{(2)}, h_{(2)}\right)\right] \sigma^{-1}\left(x_{(3)}, h_{(3)}\right) \lambda\left(\gamma\left(x_{(4)}\right) v\right) \\
& =\sum_{(x)(h)} \gamma^{-1}\left(h_{(1)}\right) \gamma^{-1}\left(x_{(1)}\right) \varepsilon\left(x_{(2)}\right) \varepsilon\left(h_{(2)}\right) \lambda\left(\gamma\left(x_{(3)}\right) v\right) \\
& =\gamma^{-1}(h) \sum_{(x)} \gamma^{-1}\left(x_{(1)}\right) \lambda\left(\gamma\left(x_{(3)}\right) v\right)=\gamma^{-1}(h) \tilde{\lambda}(v) . \quad
\end{aligned}
$$

THEOREM 6.4. Let $H$ be a finite dimensional semisimple cocommutative Hopf algebra, and $A \#_{\sigma} H$ a crossed product with $\sigma$ invertible. If $W \subseteq V$ are (left) $A \#_{\sigma} H$ modules so that $W$ has an $A$-complement in $V$, then $W$ has an $A \#_{\sigma} H$ complement in $V$.

Proof. Since $H$ is finite dimensional and semisimple, there exists a left integral $x \in H$ with $\varepsilon(x) \neq 0[25$, p. 103]. Consequently $H$ contains a left integral $e$ so that $\varepsilon(e)=1$.

Since $W$ has an $A$-module complement in $V$, there exists an $A$-module projection $\pi: V \rightarrow W$.

Since $H$ is cocommutative, $\gamma$ is invertible and $A \#_{\sigma} H=(A \# 1) \gamma^{-1}(H)$ by Lemma 6.2. We may therefore apply Lemma 6.3, to conclude that the map

$$
\tilde{\pi}(v)=\sum_{(e)} \gamma^{-1}\left(e_{(1)}\right) \pi\left(\gamma\left(e_{(2)}\right) v\right)
$$

is an $A \#_{\sigma} H$-module map.

We claim that $\tilde{\pi}$ is also a projection of $V$ onto $W$. For if $w \in W$, then $\pi(w)=w$, and thus

$$
\tilde{\pi}=\sum_{(e)} \gamma^{-1}\left(e_{(1)}\right) \pi\left(\gamma\left(e_{(2)}\right) w\right)=\sum_{(e)} \gamma^{-1}\left(e_{(1)}\right) \gamma\left(e_{(2)}\right) w=\varepsilon(e) w=w .
$$

Thus $W$ has an $A \#_{\sigma} H$-complement, namely $\operatorname{Ker} \tilde{\pi}=(I-\tilde{\pi}) V$.

We can now obtain our version of Maschke's theorem.

COROLLARY 6.5. Let $H$ be a finite dimensional semisimple cocommutative Hopf algebra, and $A \#_{\sigma} H$ a crossed product with $\sigma$ invertible.

(1) Let $V$ be a (left) $A \#_{\sigma} H$-module. If $V$ is completely reducible as an $A$ module, then $V$ is completely reducible as an $A \#_{\sigma} H$-module.

(2) If $A$ is semisimple Artinian, so is $A \#_{\sigma} H$. 
ProOF. (1) is immediate from Theorem 6.4. Now (2) follows from (1), using the fact that an algebra is semisimple Artinian if and only if every module is completely reducible.

We next consider semisimplicity, when $A$ is not Artinian. For twisted products, the standard method of induced modules works.

COROLlaRY 6.6. Let $H$ be finite dimensional and semisimple, and $A \#_{\sigma} H$ a crossed product with $A$ a semisimple algebra. Then $A \#_{\sigma} H$ is semisimple in the following cases.

(1) $\sigma$ is trivial and the action of $H$ is inner,

(2) $\sigma$ is invertible, $H$ is cocommutative, and the action of $H$ is trivial.

Proof. From Corollary 5.5, in the case $\sigma$ is trivial and the action is inner we know that $A \# H \cong A_{t}[H]$. Thus in both cases (1) and (2), we are actually dealing with twisted products.

Now for any twisted product $A_{\sigma}[H]$, and any (left) $A$-module $V$, we form the induced $A_{\sigma}[H]$-module $\bar{V}=A_{\sigma}[H] \otimes_{A} V$. Since the elements of $A$ commute with the elements of $H, \bar{V} \cong \sum_{i=1}^{n} \oplus V$ as an $A$-module, where $\operatorname{dim}_{k} H=n$. Thus if $V$ is a completely reducible $A$-module, so is $\bar{V}$.

An algebra $A$ is semisimple if and only if there exists a faithful completely reducible $A$-module, say $V$. Constructing $\bar{V}$ as above, $\bar{V}$ is certainly faithful as an $A_{\sigma}[H]$-module. It is also completely reducible as an $A_{\sigma}[H]$-module: use Proposition 6.1 in case (1) and Corollary 6.5 in case (2). Thus $A_{\sigma}[H]$ is semisimple since it has a faithful completely reducible module.

The foregoing argument does not extend to $A \#_{\sigma} H$ when the action of $H$ on $A$ is not inner, since it is false in general that, if $V$ is a completely reducible $A$-module, $\bar{V}=\left(A \#_{\sigma} H\right) \otimes_{A} V$ is completely reducible as an $A$-module even when $\sigma$ is trivial, as we show in our next example.

EXAMPLE 6.7. Let $G$ be a finite group. Let $A=k G, H=(k G)^{*}, V$ the trivial one dimensional $G$-module $k$, and form $\bar{V}=(A \# H) \otimes_{A} V$. Let $\left\{p_{x}: x \in G\right\}$ be the basis of $H$ dual to the basis $G$ of $A$. We have

$$
\left(1 \# p_{y}\right)(x \# 1)=x \# p_{x^{-1} y}
$$

for $x, y \in G$, which shows that $A \# H$ is a free right $A$-module with basis $\left\{1 \# p_{x}: x \in G\right\}$. Thus we may identify $\bar{V}$ with $H: p_{x} \leftrightarrow\left(1 \# p_{x}\right) \otimes_{A} 1$. Moreover, the left action of $A$ on $\bar{V}$ corresponds to the action of $A$ on $H$ given by $x \cdot p_{y}=p_{x y}$. In fact,

$$
(x \# 1)\left(1 \# p_{y}\right) \otimes_{A} 1=\left(1 \# p_{x y}\right)(x \# 1) \otimes_{A} 1=\left(1 \# p_{x y}\right) \otimes_{A} 1 .
$$

This shows that as an $A$-module, $\bar{V}$ is isomorphic to $A$ under the left regular representation of $A$ on itself. Now if the characteristic of $k$ divides the order of $G$, $k G$ is not completely reducible as a left $G$-module.

Despite this example, it $i s$ always true that $A$ semisimple implies $A \#(k G)^{*}$ is semisimple, using other methods $[\mathbf{4}]$.

We are able to show that $A$ semiprime implies $A \#_{\sigma} H$ semiprime in the two cases of Corollary 6.6. Our method is to use the "primitivity machine" of Lorenz and Passman [13] to reduce to the semisimple situation. 
If $R$ is any ring with 1 , let $\prod R$ denote the complete direct product of copies of $R$ indexed over the natural numbers, and let $\sum R$ denote the direct sum. Now define

$$
\hat{R}=\prod R / \sum R
$$

and denote the elements of $\prod R$ by $\left(a_{n}\right)$.

LEMMA 6.8 [13]. The following are equivalent:

(1) $S$ is semiprime,

(2) $\hat{S}$ has no nonzero nil ideals,

(3) $\hat{S}$ is semiprime.

The next lemma was proved in $[\mathbf{1 3}]$ for $H=k G$.

Lemma 6.9. Let $A \#_{\sigma} H$ be a crossed product, with $H$ finite dimensional. Then

$$
\widehat{A \#_{\sigma} H} \cong \hat{A} \#_{\sigma} H \text {. }
$$

Proof. The (weak) action of $H$ on $A$ can be extended to $\prod A$ by letting it act on each component; since $\sum A$ is stable under this action, the induced action of $H$ on $\hat{A}$ is well defined. Similarly $\sigma: H \times H \rightarrow A$ may be extended to $\bar{\sigma}: H \times H \rightarrow \hat{A}$ by defining

$$
\bar{\sigma}(h, k)=(\sigma(h, k), \ldots, \sigma(h, k), \ldots)+\sum A .
$$

Clearly $\hat{A} \#_{\sigma} H$ is a crossed product.

The fact that $\widehat{A \#_{\sigma} H} \cong \hat{A} \#_{\sigma} H$ follows by exactly the same argument used by Lorenz and Passman [13], except that a fixed basis $\left\{h_{1}, \ldots, h_{n}\right\}$ of $H$ is used instead of the group elements $\{g \mid g \in G\}$.

THEOREM 6.10. Let $H$ be finite-dimensional and semisimple, and $A \#_{\sigma} H a$ crossed product with $A$ a semiprime algebra. Then $A \#_{\sigma} H$ is semiprime in the following two cases.

(1) $\sigma$ is trivial and the action of $H$ is inner,

(2) $\sigma$ is invertible, $H$ is cocommutative, and the action of $H$ is trivial.

Proof. We form $\widehat{A \#_{\sigma} H}$; by Lemma 6.9 , it suffices to show $\widehat{A \#_{\sigma} H}$ is semiprime; by Lemma 6.9 , this is equivalent to showing $\hat{A} \#_{\sigma} H$ is semiprime.

Now since $A$ is semiprime, $\hat{A}$ has no nil ideals by Lemma 6.8. Thus the polynomial ring $\hat{A}[x]$ is semisimple by Amitsur's theorem. By Corollary 6.6, $\hat{A}[x] \#_{\sigma} H$ is also semisimple, where $\hat{A}[x] \#_{\sigma} H$ makes sense by letting $H$ act trivially on $x$. But then $\hat{A}[x] \#_{\sigma} H=\left(\hat{A} \#_{\sigma} H\right)[x]$ is semisimple. Thus $\hat{A} \#_{c} H$ has no nilpotent ideals, so is semiprime.

\section{REFERENCES}

1. J. Bergen and M. Cohen, Actions of commutative Hopf algebras, Bull. London Math. Soc. 18 (1986), 159-164.

2. R. J. Blattner and S. Montgomery, A duality theorem for Hopf module algebras, J. Algebra 95 (1985), 153-172.

3. M. Cohen and D. Fishman, Hopf algebra actions, J. Algebra 100 (1986), 363-379.

4. M. Cohen and S. Montgomery, Group-graded rings, smash products, and group actions, Trans. Amer. Math. Soc. 282 (1984), 237-258. 
5. E. C. Dade, Group graded rings and modules, Math. Z. 174 (1980), 241-262.

6. J. Dixmier, Enveloping algebras, North-Holland, Amsterdam, 1977.

7. Harish-Chandra, On representations of Lie algebras, Ann. of Math. (2) 50 (1949), 900-915.

8. I. Kaplansky, Hopf algebras, Univ. of Chicago Lecture Notes, 1975.

9. V. K. Kharchenko, The actions of groups and Lie algebras on noncommutative rings, Russian Math. Surveys 35:2 (1980), 77-104.

10. M. Landstad, J. Phillips, I. Raeburn and C. Sutherland, Representations of crossed products by coactions and principal bundles (to appear).

11. R. Larson and M. E. Sweedler, An associative orthogonal bilinear form for Hopf algebras, Amer. J. Math. 91 (1969), 75-94.

12. M. Lorenz, Primitive ideals in crossed products and rings with finite group actions, Math. Z. 158 (1978), 285-294.

13. M. Lorenz and D. S. Passman, Two applications of Maschke's theorem, Comm. Algebra 8 (1980), 1853-1866.

14. __ Prime ideals in group algebras of polycyclic-by-finite groups, Proc. London Math. Soc. 43 (1981), 520-543.

15. W. Magnus, A. Karrass, and D. Solitar, Combinatorial group theory, Interscience, New York, 1966.

16. S. Montgomery, Fixed rings of finite automorphism groups of associative rings, Lecture Notes in Math., vol. 818, Springer-Verlag, Berlin, 1980.

17.,$X$-inner automorphisms of filtered algebras, Proc. Amer. Math. Soc. 83 (1981), 263-268.

18. S. Montgomery and D. S. Passman, Crossed products over prime rings, Israel J. Math. 31 (1978), 224-256.

19. _,$X$-inner automorphisms of group rings, Houston J. Math. 7 (1981), 395-402.

20. $\ldots$ _. $X$-inner automorphisms of crossed products and semiinvariants of Hopf algebras, Isreal J. Math. (to appear).

21. Y. Nakagami and M. Takesaki, Duality for crossed products of von Neumann algebras, Lecture Notes in Math., vol. 731, Springer-Verlag, Berlin, 1979.

22. G. Pedersen, $C^{*}$-algebras and their automorphism groups, Academic Press, London, 1979.

23. R. Steinberg, Complete sets of representatives of algebras, Proc. Amer. Math. Soc. 13 (1962), 746-747.

24. M. E. Sweedler, Cohomology of algebras over Hopf algebras, Trans. Amer. Math. Soc. 133 (1968), 205-239.

25. __ Hopf algebras, Benjamin, New York, 1969.

Department of Mathematics, University of CAlifornia, Los ANGeles, CaliFORNIA 90024

Department of Mathematics, Ben Gurion University of the Negev, BeerSHEVA, ISRAEL

Department of Mathematics, University of Southern California, Los ANGELES, CALIFORNIA 90089-1113 